\title{
Kinetics of gelation and universality
}

\author{
R M Ziff $\dagger$, M H Ernst $\ddagger$ and E M Hendriks $\ddagger$ \\ † Department of Chemical Engineering, Dow Building, University of Michigan, Ann \\ Arbor, Michigan 48109, USA \\ † Institute for Theoretical Physics, Princetonplein 5, State University, 3508 TA Utrecht, \\ The Netherlands
}

Received 17 December 1982

\begin{abstract}
The exact solution (size distribution $c_{k}(t)$ and moments $M_{n}(t)$ ) of Smoluchowski's coagulation equation ( $\mathrm{S}$-model) and of a modified equation ( $\mathrm{F}$-model) with a coagulation rate $K_{i j}=i j$ for $i$ - and $j$-clusters is obtained for arbitrary $c_{k}(0)$ in the sol $\left(t<t_{c}\right)$ and gel $\left(t>t_{c}\right)$ phases, where $t_{c}$ is the gel point. The behaviour of $c_{k}(t)$ and $M_{n}(t)$ is given for $k \rightarrow \infty, t \rightarrow \infty$ and $t \rightarrow t_{\mathrm{c}}$. The critical exponents, critical amplitudes and scaling function that characterise the singularities near the non-equilibrium phase transition are calculated. For short-range $c_{k}(0)$ (i.e. all $M_{n}<\infty$ ) the F-model belongs to the universality class of classical gelation theories and of bond percolation on Cayley trees; the $\mathrm{S}$-model does not.
\end{abstract}

\section{Introduction}

The kinetics of polymerisation, and in particular of gelation, has recently attracted considerable attention. Our main concern here is to present a kinetic description of the gelation transition, starting from arbitrary initial distributions. This will be done for the gelation models of Flory (1953) and Stockmayer (1943). The hypotheses of scaling and of universality (the latter refers here to independence of the initial size distribution) will be investigated for critical exponents and ratios of critical amplitudes (Aharony 1980, Stauffer et al 1982), describing the singularities occurring in this kinetic phase transition.

In the statistical theory of Flory and Stockmayer for branched polymers, and in the statistical mechanical methods for the comparable bond percolation problem on Bethe lattices (de Gennes 1976, Stauffer 1976), one always assigns equilibrium weights to the allowed distribution over cluster sizes, corresponding to the given value of the conversion $\alpha$ (i.e. concentration of bonded reactive groups, $0<\alpha<1$ ). The equilibrium weights of the standard theories correspond in a kinetic description to the very special monodisperse initial distribution, $c_{k}(0)=\delta_{k 1}$ for which the kinetic results are identical to the standard ones (Ziff and Stell 1980).

In view of recent experimental tests of the kinetic theories on polymerisation (von Schulthess et al 1980, Schmidt and Burchard 1981) it is also of interest to have theoretical predictions for general initial distributions. It will turn out that all physically relevant properties are determined by the first few moments of the initial distribution. Hence the cumbersome task of preparing monodisperse initial distributions can be avoided.

The basic equation is Smoluchowski's coagulation equation (Drake 1972, Cohen and Benedek 1982) which describes the evolution of a system of particles which are 
continuously growing as a result of pairs of particles coming into contact and bonding or sticking together. Examples include the coagulation of colloidal suspensions and the formation of polymers. These systems may, in general, be described by the kinetic equation

$$
\dot{c}_{k}=\frac{1}{2} \sum_{i+j=k} \boldsymbol{K}_{i j} c_{i} c_{j}-c_{k} \sum_{j=1}^{\infty} \boldsymbol{K}_{k j} c_{i}
$$

The size distribution $c_{k}(t)(k=1,2, \ldots)$ represents the set of concentrations of finite size clusters (sol particles) with $k$ basic units. It is normalised such that $\Sigma k c_{k}(0)=$ $M(0)=1$. The gain and loss terms contain $K_{i j} c_{i} c_{j}$, representing the rate at which $i$-clusters and $j$-clusters combine to form $(i+j)$-clusters, and the sum extends over all sol particles.

The coagulation rates $K_{i j}$ in our kinetic model are determined by the statistical probabilities of bond formation and by the diffusivity of clusters. Here the latter effect, discussed by Cohen and Benedek (1982) and Hendriks et al (1983), will be neglected.

The coagulation rates depend upon the details of the physical process being considered. In the Flory-Stockmayer theory for branched polymers a $k$-cluster will contain exactly $(k-1)$ bonds, and $s_{k}=(\bar{f}-2) k+2$ free reactive groups, because it is assumed that only tree-like structures can form with no intra-molecular bonding allowed $\uparrow$. Since all free groups are assumed to be equally reactive the coagulation rate is $K_{i j}=s_{i} s_{j}$, and the kinetic equation for the sol particles can be written as

$$
\dot{c}_{k}=\frac{1}{2} \sum_{i+j=k} s_{i} s_{j} c_{i} c_{j}-\mu_{s} s_{k} c_{k}
$$

where $k$-clusters are lost due to bonding with all available reactive groups $\mu_{\mathrm{s}}$ in the sol:

$$
\mu_{\mathrm{s}}(t)=\sum_{k=1}^{\infty} s_{k} c_{k}(t)
$$

A fundamental property of (1.2) $\ddagger$ is the conservation of total mass of sol particles, $M(t)=\Sigma k c_{k}(t)=1$, for times smaller than some critical time $t_{\mathrm{c}}$ (gel point). For $t \geqslant t_{\mathrm{c}}$ there is a loss of mass $(\dot{M} \neq 0)$ to $k=\infty$. This loss of mass is interpreted as the formation of the gel (infinite cluster), and the gel fraction

$$
G(t)=1-M(t)
$$

represents the probability of a unit to belong to the infinite cluster. Past the gel point the concentration of $k$-clusters will decrease due to reactions with all available reactive groups, $\mu(t)$, not only on sol particles $\mu_{\mathrm{s}}(t)$, but also on the gel, $\mu_{\mathrm{g}}(t)$, where

$$
\mu(t)=\mu_{\mathrm{s}}(t)+\mu_{\mathrm{g}}(t) .
$$

The latter loss mechanism is, however, absent in (1.2) and one may modify (1.2) past the gel point to account for mass loss to the gel. This may be done by replacing $\mu_{\mathrm{s}}$ in (1.2) by $\mu$, and supplying a rate equation for $\mu_{g}(t)$, which depends on the assumed interactions between sol and gel. Dusek (1979) and Ziff and Stell (1980) propose one model (to be referred to as the F-model) in which the rate equation for the total

\footnotetext{
$\dagger$ Note the equivalence with bond percolation on a Bethe lattice or Cayley tree of coordination number $\bar{f}$. $\ddagger$ Possible functional forms of $K_{i j}$ yielding a gelation transition have been discussed by Ernst et al (1982) and the corresponding (non-classical) critical exponents have been calculated.
} 
number of free groups, $\mu(t)$, is the same before $\left(\mu=\mu_{\mathrm{s}}\right)$ and after $\left(\mu=\mu_{\mathrm{s}}+\mu_{\mathrm{g}}\right)$ the gel point. From the present kinetic equation one easily derives that the relevant equation in the F-model is $\dot{\mu}=-\mu^{2}$. Ziff and Stell also show that the above F-model with monodisperse initial conditions corresponds to Flory's classical gelation theory.

A second model (to be referred to as the S-model) with $\mu=\mu_{\mathrm{s}}$ for all times (absence of sol-gel interactions, since $\mu_{\mathrm{g}} \equiv 0$ ) corresponds to Stockmayer's classical gelation theory ${ }^{\dagger}$. This model has been solved for all times and monodisperse initial conditions by Ziff $(\bar{f}=3)$, Ziff and Stell (general $\bar{f})$, and Leyvraz and Tschudi $(1981)(\bar{f} \rightarrow \infty)$. The explicit solutions of (1.2) in the sol phase $\left(t<t_{\mathrm{c}}\right)$ for the mono-disperse initial condition have already been known for a long time and have been given by Stockmayer (1943) (general $\bar{f})$ and McLeod (1962) $(\bar{f} \rightarrow \infty)$. For general initial conditions the solution is only known for $t<t_{\mathrm{c}}$, at which time the moments, defined as

$$
M_{n}(t)=\sum_{k=1}^{\infty} k^{n} c_{k}(t)
$$

diverge for $n \geqslant 2$ (Drake 1972). The time $t_{\mathrm{c}}$ is given in terms of $M_{2}(0)$. A very different approach has been taken by Lushnikov (1978; Lushnikov et al 1981); using standard arguments (see van Kampen 1981), he constructs a master equation for the probability distribution $P\left\{c_{1} c_{2} \ldots c_{k} \ldots, t\right\}$, in which the transition probabilities are determined by the coagulation rates $K_{i j}$ in the macroscopic rate equations. Thus he is able to account ror the fluctuations in $c_{k}$. The macroscopic size distribution in Smoluchowski's equation is then an average $\bar{c}_{k}$. Close to and past the gel point the fluctuations become of macroscopic size, and effectively modify the macroscopic rate equations. In the thermodynamic limit, i.e. to leading order in an expansion in inverse powers of the volume, Lushnikov's results for the size distribution, mean cluster number and sol mass are identical to the results of the F-model, as will be shown in this paper.

In order to simplify the mathematics we consider monomers of high functionality $\bar{f} \dot{f}$, such that the number of free groups on a $k$-cluster is $s_{k} \approx \bar{f} k$, and redefine $\bar{f}^{2} t$ as the new time variable. The general form of the coagulation equation, valid in the sol and gel phase becomes:

$$
\dot{c}_{k}=\frac{1}{2} \sum_{i+j=k} i j c_{i} c_{j}-\mu k c_{k}
$$

where $\mu(t)$ represents the total number of free reactive groups in the system (in redefined units). In the sol phase

$$
\mu(t)=\mu_{\mathrm{s}}(t) \equiv \sum k c_{k}(t)=1
$$

and in the gel phase

$$
\mu(t)=\mu_{\mathrm{s}}(t)+\mu_{\mathrm{g}}(t) .
$$

In the F-model version of $(1.7), \mu(t)$ satisfies the same equation in both sol and gel phase. Thus we have by virtue of $(1.8 a) \dot{\mu}=0$ for all $t$, or

$$
\mu(t)=M(0)=1 .
$$

\footnotetext{
t A third model, proposed by Ziff and Stell (1980), coincides with the F-model in the limit of large $\bar{f}$, to which we shall restrict ourselves in this paper.

¥Von Schulthess et al (1980) have performed experiments where $\bar{f}$ is large, and Cohen and Benedek (1982) emphasise the mathematical simplifications in this limit.
} 
Note that the equation $\dot{\mu}=-\mu^{2}$ at finite $\bar{f}$ reduces in redefined units $\bar{f}^{2} t \rightarrow t$ and $\mu / \bar{f} \rightarrow \mu$ to $\dot{\mu}=0$ in the limit $\bar{f} \rightarrow \infty$.

In the S-model version of $(1.7), \mu_{\mathrm{g}}=0$, so that for all $t$ we have

$$
\mu(t)=M(t) .
$$

In order to clarify the differences between the kinetic models we briefly compare the statistical theories of Flory ( $\mathrm{F}$-model) and Stockmayer ( $\mathrm{S}$-model) in so far as is necessary for our purpose (see also Ziff and Stell 1980; Cohen and Benedek 1982, Donoghue and Gibbs 1979, Donoghue 1982).

The size distribution for a macroscopic system, specified by $M$ and $M_{0}$, the number of monomeric units and clusters (per volume, say), respectively, is found to be $c_{k}=A D_{k} \xi^{k}$, where $D_{k}$ is a combinatorial factor equal to the number of distinct ways a $k$-mer can be composed out of $k$ monomeric units, each having $\bar{f}$ equivalent reactive groups. The normalisation constant $A$ and fugacity $\xi$ are determined by prescribed values of $M_{0}\left(=A \Sigma D_{k} \xi^{k}\right)$ and $M\left(=A \Sigma k D_{k} \xi^{k}\right)$, or alternatively by values of the mass $M$ and the conversion ${ }^{\dagger} \alpha=(2 / \bar{f})\left(1-M_{0} / M\right)$. The series for $M_{0}$ and $M$, defining $\alpha(\xi)$, have a finite radius of convergence $\xi_{\mathrm{c}}$, corresponding to $\alpha_{\mathrm{c}}=$ $(\bar{f}-1)^{-1}$. The inverse function $\xi(\alpha)=\alpha(1-\alpha)^{\bar{f}-2} / \bar{f}$ is a single-valued function that reaches a maximum $\xi_{\mathrm{c}}$ at $\alpha=\alpha_{\mathrm{c}}$. Below the gel point a prescribed value of $\alpha$ fixes the fugacity $\xi\left(\xi<\xi_{\mathrm{c}}\right)$, so that the size distribution $c_{k}=A D_{k} \xi^{k}$ is identical in the Fand S-models.

In Flory's theory the value of $\alpha\left(\alpha>\alpha_{c}\right)$ is also prescribed above the gel point $\alpha_{\mathrm{c}}$. To every $\alpha$ belongs a unique value of the fugacity $\xi\left(\xi<\xi_{\mathrm{c}}\right)$ through the inverse function $\xi(\alpha)$. The size distribution of sol particles is $c_{k}=A D_{k} \xi^{k}$ and all moments exist. As $\alpha$ increases from $\alpha_{c}$ to unity, the increasing number of reactive groups on the gel causes the fugacity in the coexisting sol and gel phase to decrease to zero as $\alpha \rightarrow 1$. As already discussed by Flory and Stockmayer, the gel can form cycles in the F-model, since $\alpha$ can exceed the value of $2 / \bar{f}$ (which would be its maximum value if there were no crosslinks in the gel) reached when all monomers are coalesced into a single macroscopic cluster (gel) without crosslinks.

A macroscopic structure containing a significant fraction of all reactive groups in the system can form crosslinks, because the probability that a given group reacts with another unit on the same structure is significant (Falk and Thomas 1974). The Flory theory of gelation can also be derived (see de Gennes 1979, Ziman 1979) from the theory of branching processes (Feller 1968). Stockmayer's theory above the gel point is very different. If $\alpha$ is increased by increasing the mass density of sol particles, the system will respond by transforming the added mass into gel in such a manner that the fugacity remains constant, $\xi=\xi_{\mathrm{c}}$. This implies that the growing gel fraction with its increasing number of reactive groups is not allowed to react with the sol, as otherwise the fugacity would necessarily decrease. The size distribution in the S-model is given by $c_{k}=A D_{k} \xi_{\mathrm{c}}^{k}$ with $A \propto M_{\text {sol }}$ (through (1.12); the relative amounts of $k$-mers and mean cluster size $M_{\text {sol }} / M_{0}$ in the sol remain fixed at their critical values, all moments $M_{n}$ with $n \geqslant 2$ are divergent and the fraction of reacted groups in the gel remains constant, i.e. $\alpha_{\text {gel }}=2 / \bar{f}$ (no crosslinks).

The phase transition in the S-model is similar to Bose-Einstein condensation. The particles condensed in the ground state correspond to the gel, the excited particles

$\div$ In the corresponding bond percolation problem on a Bethe lattice, $\alpha$ is the probability for the presence of a bond. This model corresponds to the F-model of gelation. 
to the sol, and the fugacity of the coexisting phases keeps the constant value $\xi=\xi_{\text {c }}$. The previous discussion shows that the kinetic F-model will correspond to Flory's theory and the kinetic S-model to Stockmayer's. Past the gel point, in both models there will be a single macroscopic cluster (gel) of size $k_{0} \cong M_{\text {gel }}$, so that $c_{k_{0}} \cong 1 / M$. It contains a total number of reactive groups equal to $\mu_{\mathrm{g}}=s_{\mathrm{k}_{0}} c_{\mathrm{k}_{0}} \propto M_{\mathrm{gel}} / M=G$. In the thermodynamic limit of the kinetic F-model the macroscopic cluster will contribute a non-vanishing term $\mu_{\mathrm{g}} \propto G$ to the total number of reactive groups $\Sigma s_{k} c_{k}$, appearing in the loss term of the kinetic equation. In the S-model the contribution of the macroscopic cluster has disappeared from the loss term. An essential difference between the kinetic F-and S-models is that the gel interacts with itself (cyclisation) in the F-model, whereas it does not in the S-model, as was shown by Ziff and Stell. Recently Donoghue and Gibbs (1979; Donoghue 1982) have presented a statistical theory of gelation for a finite system containing a total of $M$ monomeric units in which the assumption of acyclic structures is strictly maintained. Donoghue shows that the mass distribution for finite large $k(k<M)$ and $\alpha>\alpha_{\mathrm{c}}$ has two well separated peaks, corresponding to sol and gel. In the limit as $M \rightarrow \infty$ at constant extent of reaction $\alpha$ the size distribution and all its moments approach the values derived by Stockmayer.

The program of this paper will be to solve the kinetic equation (1.7) for the Fand S-models analytically for all $t$ and arbitrary initial conditions.

In $\& 2$ we determine the generating function of the $c_{k}(t)$, and discuss the properties of the sol mass in the sol and gel phase. Extensive use will be made of the graphical analysis of a plot of the generating function, as first introduced by Ziff and Stell. This helps to illustrate many properties of the solution, such as asymptotic behaviour of $c_{k}(t)$ and $M_{n}(t)$, near both $t=t_{\mathrm{c}}$ and $t=\infty$.

In $\& 3$ Lagrange's expansion is used to derive general expressions for $c_{k}(t)$ and $M_{n}(t)$, valid for all $k, n$ and $t$. The results for the moments are analysed in the vicinity of the gel point for different classes of initial distributions: namely short-range $c_{k}(0)$ (exponentially cut off) and long-range $c_{k}(0)$ (with algebraic tails). The appropriate critical exponents and amplitudes are calculated, and tested for the hypotheses of scaling and universality.

In $\S 4$ asymptotic results for $c_{k}(t)$ are derived: for $k \rightarrow \infty$ and fixed $t$; for $t \rightarrow \infty$ and fixed $k$, and for the (coupled) scaling limit with $k \rightarrow \infty$ and $t \rightarrow t_{\mathrm{c}}$. Again the predictions of scaling and universality are tested. The final conclusions are summarised in $\S 5$.

Before concluding this section we briefly introduce the critical exponents and amplitudes, mainly following the notation of Aharony (1980), and quote the universal values for exponents and amplitude ratios in the classical bond percolation problem needed for a comparison with our results. In a non-equilibrium description of the gelation transition the distance to the gel point $t_{\mathrm{c}}$ is measured in the dimensionless time $\theta=\left(t / t_{\mathrm{c}}-1\right)$. The quantities of interest are: the gel fraction (order parameter), decreasing for $t \downarrow t_{\mathrm{c}}$ as

$$
G(t) \simeq B \theta^{\beta} ;
$$

the singular part of the mean cluster number $M_{0}$ (as defined in (1.6)) behaving for $t \rightarrow t_{\mathrm{c}}$ as

$$
\left\{M_{0}(t)\right\}_{\text {sing }} \simeq A^{ \pm}|\theta|^{2-\alpha}
$$

It is the analogue of the free energy in thermodynamic phase transitions. 
The moments $M_{2}, M_{3} \ldots$, as defined in (1.6), diverge for $t \rightarrow t_{\mathrm{c}}$ as

$$
M_{2}(t) \simeq C^{ \pm}|\theta|^{-\gamma}, \quad M_{3}(t) \simeq D^{ \pm}|\theta|^{-\gamma-1 / \sigma},
$$

where the superscript $+(-)$ refers to the sol (gel) phase with $\theta<0(\theta>0)$. Here $M_{2}(t)$ is the analogue of the order parameter susceptibility, and

$$
k_{\xi}=M_{3}(t) / M_{2}(t)
$$

is a measure for the critical cluster size, related to the correlation length $\xi$ by the relation $\xi \sim k_{\xi}^{\rho}$, where $\rho$ is a geometric exponent (see Stauffer et al 1982) that will not be considered here. Introduction of a 'ghost' field $H$ yields the analogue of the critical 'isotherm' (Aharony 1980):

$$
\left\{f\left(-H, t_{\mathrm{c}}\right)\right\}_{\mathrm{sing}}=\left\{\sum k c_{k}\left(t_{\mathrm{c}}\right) \mathrm{e}^{-k H}\right\}_{\text {sing }} \simeq 1-E H^{1 / \delta} .
$$

An important concept in modern theories of phase transitions is the scaling hypothesis. It states that $c_{k}(t)$ for large clusters in the close vicinity of the gel point has the form

$$
c_{k}(t) \simeq q_{0} k^{-\tau} \Phi\left(k|\theta|^{1 / \sigma}\right),
$$

where the critical amplitude $q_{0}$ and scaling function $\Phi(x)$ are defined such that $\Phi(0)=1$. It follows from the scaling hypothesis that all exponents $\alpha, \beta, \gamma$ and $\delta$ can be expressed in $\tau$ and $\sigma$.

The classical values for the exponents in the bond percolation problem are obtained from Aharony by taking $\varepsilon=0$ in his results, corresponding to dimensionality $d=6$ :

$\alpha=-1, \quad \beta=1, \quad \gamma=1, \quad \delta=2, \quad \sigma=\frac{1}{2}, \quad \tau=\frac{5}{2}$.

The corresponding values for the universal ratios of critical amplitudes $t$

$$
\begin{gathered}
A^{+} / A^{-}=-\frac{1}{5}, \quad C^{+} / C^{-}=D^{+} / D^{-}=1, \quad\left(A^{+}+A^{-}\right) C^{+} / B^{2}=\frac{1}{6}, \\
C^{+} B / E^{2}=C^{+} B / 4 \pi q_{0}^{2}=1,
\end{gathered}
$$

can be deduced from Aharony's equations (1.6)-(1.10) and (7.2), using the relation $\xi=\left(k_{\xi}\right)^{\rho}$. In (1.18) the sum $\left(A^{+}+A^{-}\right)$(which equals $\left.-4 A^{+}\right)$is convenient for later purposes.

In our non-equilibrium theory of phase transitions quantities are called universal if their values are independent of the initial state of the system.

\section{General solution}

\subsection{Generating functions}

In this section we solve the initial value problem for the coagulation equation

$$
\dot{c}_{k}=\frac{1}{2} \sum_{i+j=k} i j c_{i} c_{j}-\mu k c_{k}
$$

$\div$ Note that exponents and amplitude ratios are independent of the coordination number $\bar{f}$ of the Bethe lattice. 
where

$$
\mu(t)= \begin{cases}1 & (\text { F-model }) \\ M(t) & (\text { S-model }) .\end{cases}
$$

We further determine the mass of sol particles $M(t)$, present a graphical analysis of the solution, and discuss some further properties of sol mass and gel fraction, as well as some examples.

The time evolution of the size distribution $c_{k}(t)$ for a given initial distribution $c_{k}(0)$, subject to the normalisation

$$
M(0)=\sum_{k=1}^{\infty} k c_{k}(0)=1,
$$

can be analysed best in terms of generating functions:

$$
g(x, t)=\sum_{k=1}^{\infty} c_{k}(t) \mathrm{e}^{k x}, \quad f(x, t)=g_{x}(x, t)=\sum_{k=1}^{\infty} k c_{k}(t) \mathrm{e}^{k x},
$$

where the subscript $x$ denotes a partial derivative with respect to $x$. The moments, if they exist, can be obtained from

$$
g(x, t)=\sum_{n=0}^{\infty} M_{n} x^{n} / n !
$$

or from a similar relation for $f=g_{x}$, yielding

$$
M_{n}(t)=\left[(\partial / \partial x)^{n} g(x, t)\right]_{x=0}=\left[(\partial / \partial x)^{n-1} f(x, t)\right]_{x=0} .
$$

The initial values of these functions are given by

$$
\begin{gathered}
g(x, 0)=\sum_{k=1}^{\infty} c_{k}(0) \mathrm{e}^{k x}=v(x), \quad f(x, 0)=\sum_{k=1}^{\infty} k c_{k}(0) \mathrm{e}^{k x}=v^{\prime}(x) \equiv u(x), \\
M_{n}(0)=v^{(n)}(0)=u^{(n-1)}(0) .
\end{gathered}
$$

The series are convergent for all $x \leqslant 0$, since $u(0)=1$ on account of (2.3) and a superscript $n$, as in $v^{(n)}$, denotes the $n$th derivative. Multiplying (2.1) with $k \mathrm{e}^{k x}$ and summing over $k$ yields a quasilinear partial differential equation for $f(x, t)$ :

$$
f_{1}=f_{x}(f-\mu) \text {. }
$$

Subscripts again denote partial derivatives. It can be solved by introducing the inverse function $x=X(f, t)$. Using $f_{x}=1 / X_{f}$ and $f_{t}=-X_{t} / X_{f}$ we find a simple equation $X_{t}=$ $-f+\mu$. Its solution corresponding to the initial condition $f(x, 0)=u(x)$ can be written in two equivalent forms:

$$
x=u^{-1}(f)-t f+\int_{0}^{t} \mathrm{~d} \tau \mu(\tau), \quad f=u\left(x+t f-\int_{0}^{t} \mathrm{~d} \tau \mu(\tau)\right),
$$

where $u^{-1}(f)=x$ is the inverse function of $f=u(x)$. For later purposes it is also convenient to have the solution in parametric form:

$$
x=s-t u(s)+\int_{0}^{t} \mathrm{~d} \tau \mu(\tau), \quad f(x, t)=u(s) .
$$


These equations enable us to calculate $g(x, t)$ by means of $(2.4)$ :

$$
g(x, t)=\int_{-\infty}^{x} \mathrm{~d} x^{\prime} f\left(x^{\prime}, t\right)=\int_{-\infty}^{s} \mathrm{~d} s^{\prime} u\left(s^{\prime}\right)\left[1-t u^{\prime}(s)\right]=v(s)-\frac{1}{2} t u^{2}(s)
$$

where $\mathrm{d} x^{\prime} / \mathrm{d} s^{\prime}$ follows from $(2.10 a)$.

The above equations implicitly determine $f(x, t)$ and $g(x, t)$ for a given initial distribution. Once $\mu(t)$ in $(2.2)$ is given, all $c_{k}(t)(k=1,2, \ldots)$ and all $M_{n}(t)$ $(n=0,1,2, \ldots$ ) can be deduced from the behaviour of the generating functions around $x=-\infty$ and $x=0$ respectively. In principle one has to determine $s(x, t)$ from $(2.10 a)$ and insert the result in $(2.10 b)$ to obtain $f$ as a function of $x$ and $t$, where the latter quantity occurs only parametrically.

\subsection{Sol mass in the F-model}

To calculate the sol mass $M(t)=f(0, t)$, one has to determine $s(0, t)$. Its value will be different in the F-and S-models. In the F-model (where $\mu(t)=1$ )

$$
s(0, t)=t\{u[s(0, t)]-1\}
$$

This equation has two solutions for all $t: s_{\mathrm{a}}(t)=0$ and $s_{\mathrm{b}}(t)$, provided the initial distribution $u(s)$ is regular for $s<s_{0}\left(s_{0}>0\right)$ and $u\left(s_{0}\right)=\infty$. The graphical construction of the roots is shown in figures $1(a)$ and $2(a)$. They are found as the intersection points of $f=u(x)$ (broken curve) and $f=t^{-1} x+1$ (straight line). The property $u\left(s_{0}\right)=\infty$ guarantees the existence of two roots for arbitrarily small $t \dagger$. The two solutions correspond to different branches of $f(x, t)$, of which the corresponding values of $f(0, t)$ are

$$
M_{\mathrm{a}}(t)=u\left(s_{\mathrm{a}}\right)=u(0)=1, \quad M_{\mathrm{b}}(t)=u\left(s_{\mathrm{b}}\right) .
$$

Having constructed $s_{\mathrm{a}}$ and $s_{\mathrm{b}}$, one can read off the values of $M_{\mathrm{a}}$ and $M_{\mathrm{b}}$ in figures $1(a)$ and $2(a)$. Which solution represents the physical mass depends upon the value of $t$. The physical branch of $f(x, t)$ approaches $c_{1}(t) \mathrm{e}^{x}$ as $x \rightarrow-\infty$ (see (2.4)), and is a monotonically increasing function of $x$ (all $c_{k}>0$ ). Thus, if $x \uparrow 0$ on the physical branch, $s \uparrow \zeta \equiv \min \left(0, s_{b}\right)$. Due to the convexity of $u(s), s_{\mathrm{b}}$ is always decreasing with time. For small $t$ we have $s_{\mathrm{b}}>0$, hence $\zeta=0$ (see figure $1(a)$ ). At the time $t=t_{\mathrm{c}} \equiv 1 / u^{\prime}(0)$ the curves $f=t^{-1} x+1$ and $f=u(x)$ are tangent, and the two roots coincide (see figure 3 ). At a still later time, $s_{\mathrm{b}}<0$ and $\zeta=s_{\mathrm{b}}$ and the sol mass (now given by $M_{\mathrm{b}}$ ) starts to decrease. Asymptotically $s_{b} \rightarrow-\infty$ and $M_{b} \rightarrow 0$.

In summary: the physically relevant root of $(2.12), s(0, t)=\zeta$, in the F-model is

$$
\zeta(t)=\min \left(0, s_{\mathrm{b}}\right)= \begin{cases}0, & t \leqslant t_{\mathrm{c}} \\ s_{\mathrm{b}}, & t \geqslant t_{\mathrm{c}} .\end{cases}
$$

The mass of sol particles, $M(t)$, is given by

$$
M(t)=\min \left(1, M_{\mathrm{b}}\right)=u(\zeta)= \begin{cases}1, & t \leqslant t_{\mathrm{c}}, \\ M_{\mathrm{b}}(t), & t \geqslant t_{\mathrm{c}},\end{cases}
$$

$\div$ If $u\left(s_{0}\right)<\infty$, the second solution $s_{\mathrm{b}}$ only exists for $t>s_{0} /\left[u\left(s_{0}\right)-1\right]$, where $s_{\mathrm{b}}<s_{0}$. An example of an initial distribution with $s_{0}=\infty$ is one in which $c_{k}(0)=0$ for all $k>k_{\text {max }}$. 
and the gel point $t_{\mathrm{c}}$ is

$$
t_{\mathrm{c}}=1 / u^{\prime}(0)=1 / M_{2}(0)
$$

where (2.7) has been used. Thus, $M(t)$ is a constant before $t_{c}$ (sol phase), and decreases past $t_{\mathrm{c}}$ (gel phase). The loss of mass at $t=t_{\mathrm{c}}$ is associated with the formation of an infinite cluster or gel. It is a loss to infinity due to the cascading growth of larger and larger clusters, where the process accelerates as the clusters grow larger, since the rate is given by $K_{i j}=i j$. Past $t_{c}$ the sol mass decreases through bonding by the gel. An equivalent way of determining the mass in the F-model is to put $x=0$ in $(2.9 b)$, yielding

$$
M=u[t(M-1)] .
$$

where the physical mass is the smallest root of this equation. The gel fraction $G(t)$ defined in (1.4) is the maximal root of

$$
G=1-u(-t G)
$$

\subsection{Sol mass in the $S$-model}

In the S-model $\mu(t)=M(t)$, and the solution $s(0, t)$ of $(2.10 a)$ depends on the yet unknown $M(t)$. It must be combined with $(2.10 b)$ for $x=0$ to yield a closed equation for $M(t)$ :

$$
\begin{aligned}
& M(t)=u[s(0, t)], \\
& s(0, t)=t M(t)-\int_{0}^{t} \mathrm{~d} \tau M(t)=\int_{0}^{t} \mathrm{~d} \tau \tau \dot{M}(\tau) .
\end{aligned}
$$

This functional equation can be solved by differentiating with respect to $t$, with the result

$$
\dot{M}=t \dot{M} u^{\prime}[s(0, t)] \text {. }
$$

This equation combined with $(2.19 a)$ has two solutions for all $t$, provided $u^{\prime}\left(s_{0}\right)=\infty$ with $s_{0}>0$. The first one is the constant solution

$$
M_{\mathrm{a}}(t)=M(0)=1,
$$

corresponding to

$$
S_{\mathrm{a}}(t)=0 .
$$

The second one is

$$
M_{\mathrm{c}}(t)=u\left(s_{\mathrm{c}}\right),
$$

where $s_{\mathrm{c}}(0, t)$ is determined from

$$
1 / t=u^{\prime}\left(s_{\mathrm{c}}\right)
$$

The graphical construction of $s_{\mathrm{c}}$ and $M_{\mathrm{c}}$ in figures $1(b)$ and $2(b)$ shows that the property $u^{\prime}\left(s_{0}\right) \rightarrow \infty$ (with $s_{0}>0$ ) guarantees that the solution $s_{c}$ of $(2.22 b$ ) exists for arbitrarily small time $\dagger$. The physically relevant solution for the S-model is given by

$$
\eta \equiv \min \left(0, s_{\mathrm{c}}\right)= \begin{cases}0, & t \leqslant t_{\mathrm{c}}, \\ s_{\mathrm{c}} & t \geqslant t_{\mathrm{c}} .\end{cases}
$$

\footnotetext{
+ If $u^{\prime}\left(s_{0}\right)<\infty$, the solution $s_{\mathrm{c}}$ only exists for $t>1 / u^{\prime}\left(s_{0}\right)$ (see figure $1(a)$ ).
} 
The sol mass $M(t)$ is

$$
M(t)=\min \left(1, M_{\mathrm{c}}\right)=u(\eta)= \begin{cases}1, & t \leqslant t_{\mathrm{c}}, \\ M_{\mathrm{c}}(t), & t \geqslant t_{\mathrm{c}},\end{cases}
$$

and the gel point is again given by (2.16). The derivation closely parallels that in the F-model and will not be repeated here. Since the sol particles in this model cannot be bonded by reactive groups in the gel (see introduction), the sol mass in the S-model, $M_{\mathrm{c}}(t)$, decreases only through cascading growth. This loss mechanism acts more slowly than that in the F-model, $\left(M_{\mathrm{b}}(t)<M_{\mathrm{c}}(t)\right.$ in figures $\left.2(a, b)\right)$. The analogue of $(2.17)$ follows by inserting $(2.19 b)$ into $(2.19 a)$, and the analogue of $(2.18)$ is

$$
G(t)=1-u\left(-\int_{0}^{t} \mathrm{~d} \tau \tau \dot{G}(\tau)\right)
$$

In view of the gelation transition associated with violation of sol mass conservation, it is instructive to reconsider the usual derivation of this conservation law. The partial moment $M^{(L)}(t)=\Sigma_{k=1}^{L} k c_{k}(t)$, where $L$ is some constant, satisfies the following equation in the sol phase:

$$
\dot{M}^{(L)}=-\sum_{i=1}^{L} i^{2} c_{i} \sum_{j=L-i+1} j c_{j}
$$

as can be derived from the kinetic equation (2. ) with $\mu(t)=1$. The limit $L \rightarrow \infty$ yields the mass loss rate $\dot{M}(t)$, which is vanishing $\mathrm{l}$ less $M_{2}(t)$ diverges. The latter is the case as $t \rightarrow t_{c}$, as we shall see in $\S 3.3$.
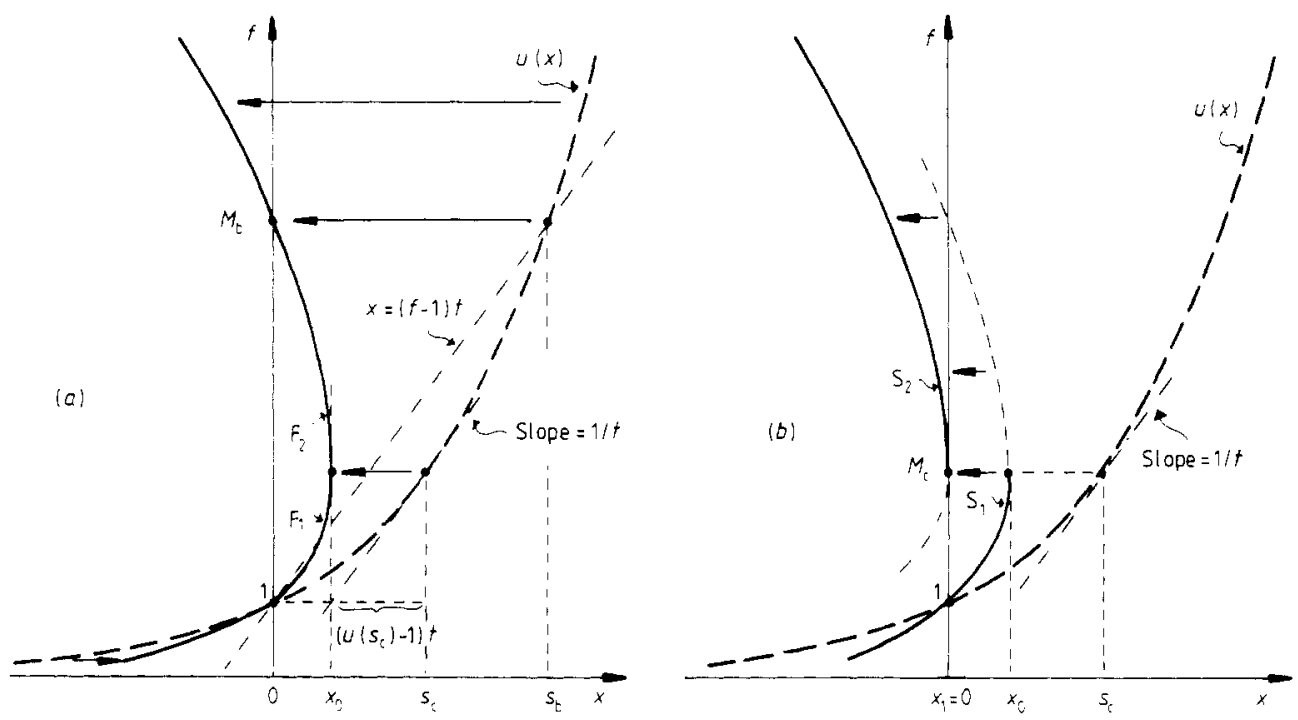

Figure 1. For $t<t_{c}$ (sol phase) and given $f(x, 0)=u(x)$ (broken curve) (a) represents two branches $\left(\mathrm{F}_{1}, \mathrm{~F}_{2}\right)$ of $f(x, t)$ in the $\mathrm{F}$-model, and $(b)$ two branches $\left(\mathrm{S}_{1}, \mathrm{~S}_{2}\right)$ of $f(x, t)$ in the S-model. The branches $F_{1}$ and $S_{1}$ with $M(t)=f(0, t)=1$ are the physical branches. 

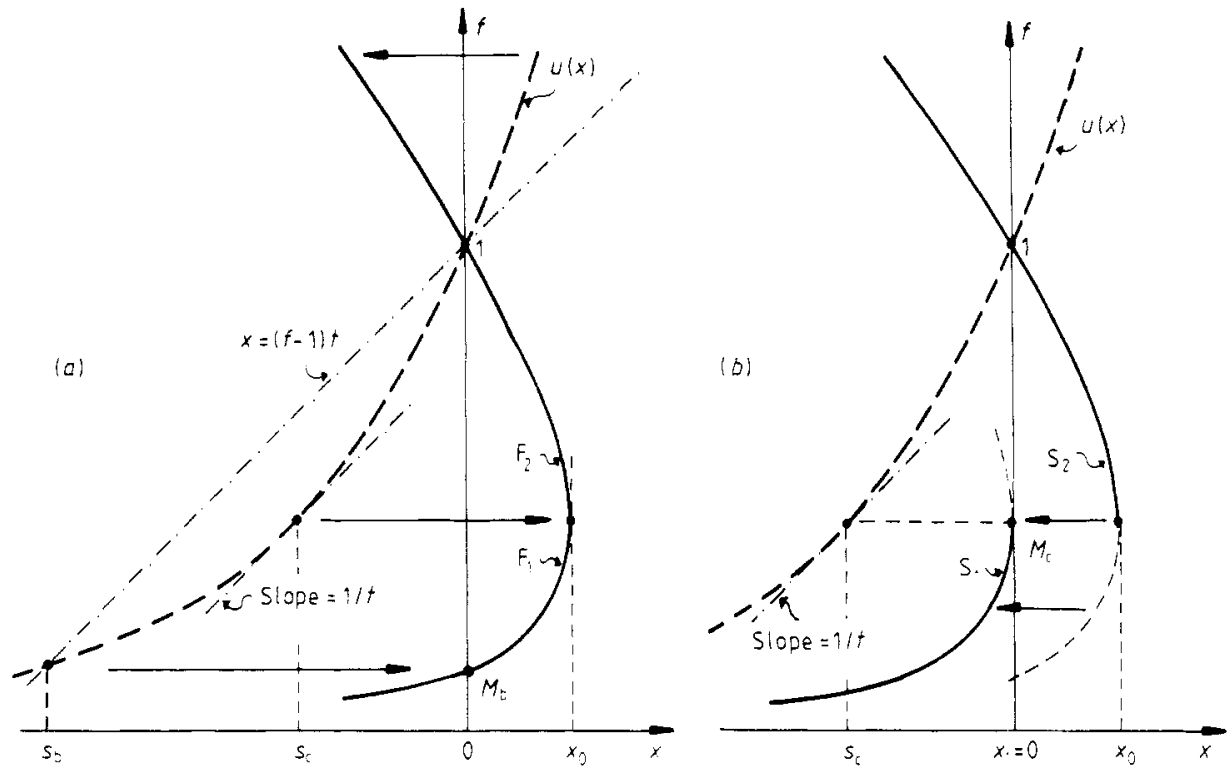

Figure 2. For $t>t_{c}$ (gel phase) and given $f(x, 0)=u(x)$ (broken curve) (a) represents two branches $\left(\mathrm{F}_{1}, \mathrm{~F}_{2}\right)$ of $f(x, t)$ in the $\mathrm{F}$-model, and $(b)$ two branches $\left(\mathbf{S}_{1}, \mathbf{S}_{2}\right)$ of $f(x, t)$ in the $\mathrm{S}$-model. The physical branches $\mathrm{F}_{1}$ and $\mathrm{S}_{1}$ yield a sol mass $M_{\mathrm{b}}(t)$ and $M_{\mathrm{c}}(t)$ respectively.

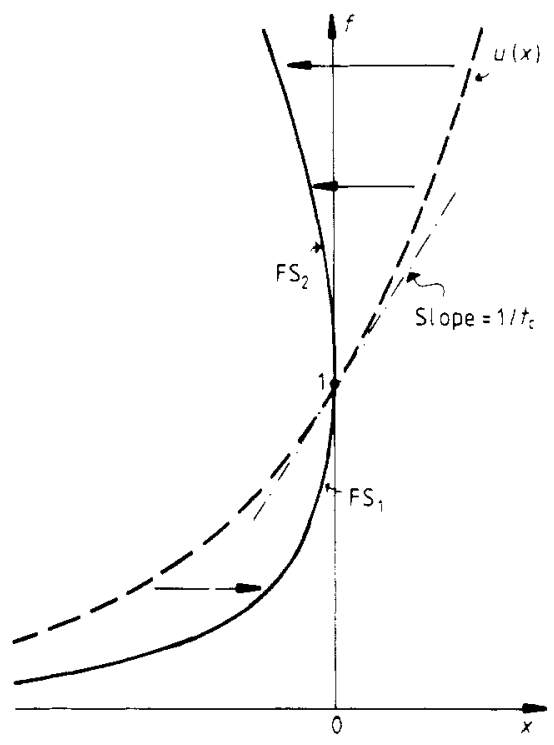

Figure 3. The full curve shows the physical $\left(\mathrm{FS}_{1}\right)$ and unphysical $\left(\mathrm{FS}_{2}\right)$ branch of $f\left(x, t_{\mathrm{c}}\right)$ at the gel point for given $f(x, 0)=u(x)$ (broken curve).

\subsection{Graphical analysis of the solution}

After the graphical construction of the physical mass in the F-and S-models, we give a graphical analysis of the solution $f(x, t)$. At the initial time $f(x, 0)=u(x)$ is represented by the broken curve, marked $u(x)$, in figures 1,2 and 3 , where (2.4) implies that 
$u(x)$ and all its derivatives approach $c_{1}(0) \mathrm{e}^{x}$ as $x \rightarrow-\infty$. First we restrict ourselves to initial conditions where $u(x)$ is regular for $x<s_{0}$ and $u\left(s_{0}\right)=\infty$ ( $s_{0}$ is positive and may be infinite).

In the F-model, where $\mu(t)=1$, the evolution of $f(x, t)$ is best exhibited using (2.9), i.e.

$$
x=u^{-1}(f)-t(f-1)
$$

For a given $f$ the corresponding $x$ changes from its initial value $u^{-1}(f)$ by an amount $-t(f-1)$. Thus, the curve of $f(x, t)$ shifts to the left for $f>1$ and to the right for $f<1$ by an amount $x=t(f-1)$, represented by the straight line in figures $1(a)$ and $2(a)$. The point $f=1$ remains stationary. The resulting plot of $f(x, t)$ is shown as the full curve, marked $\mathrm{F}_{1}, \mathrm{~F}_{2}$. Note that $f(x, t)$ has a vertical segment at $x=x_{0}$, and is a double-valued function for all $x<x_{0}$. The point $x_{0}$ is determined by the condition $(\partial x / \partial f)_{t}=0$, or because of $(2.10)$ by $(\partial x / \partial s)_{t}=1-t u^{\prime}(s)=0$. Its solution equals $s_{c}$ (see $(2.22 b)$ ), corresponding to $s_{\mathrm{c}}=u^{-1}\left[f\left(x_{0}, t\right)\right]$ or $f\left(x_{0}, t\right)=u\left(s_{\mathrm{c}}\right)=u\left[x_{0}+t u\left(s_{\mathrm{c}}\right)-t\right]$. In the last equality (2.10) has been used. Hence, we have the relation

$$
x_{0}=s_{c}-t\left[u\left(s_{c}\right)-1\right]
$$

as shown explicitly in figure $1(a)$.

The evolution of $f$ causes $u(x)$ to fold over onto itself at $x_{0}$ for all $t>0$. This folding over is the origin of the singularity that appears in $f(x, t)$ at $x_{0}$, and causes the appearance of two branches of $f(x, t)$, marked $F_{1}$ and $F_{2}$ in figures $1(a)$ and $2(a)$. One branch intersects the $f$ axis at $f(0, t)=1$ and one at $f(0, t)=M_{\mathrm{b}}(t)$. The branch $\mathrm{F}_{1}$ is the relevant one with $f(0, t)=M(t)$, since it decreases exponentially at $x=-\infty$, and determines the $c_{k}(t)$ through the expansion (2.4). The moments are determined by the derivatives of the same branch $\mathrm{F}_{1}$ at $x=0$ or $s(0, t)=\zeta$ defined in (2.14). They exist for all $t$ different from $t_{\mathrm{c}}$. At the gel point $t_{\mathrm{c}}=1 / u^{\prime}(0)$ both $x_{0}$ and $\zeta$ are zero, the singularity in $f\left(x, t_{\mathrm{c}}\right)$ is located at the origin (see figure 3) and all $M_{n}\left(t_{c}\right)$ for $n \geqslant 2$ are infinite on account of (2.6).

In the S-model, where $\mu(t)=M(t)$, the situation is somewhat different. Here we have from $(2.10 a)$

$$
x=u^{-1}(f)-t f+T
$$

with

$$
T=\int_{0}^{1} \mathrm{~d} \tau M(\tau) .
$$

For a given $f$ the corresponding $x$ changes by an amount $T-t f$, where $T$ is different for the two solutions $M_{\mathrm{a}}(t)=1$ and $M_{\mathrm{c}}(t)$, defined in (2.21) and (2.22), each yielding a different branch of $f(x, t)$ (referred to as S-branches). For the branch with $f(0, t)=$ $M_{\mathrm{a}}(1)=1$, the quantity in (2.30) equals $T_{\mathrm{a}}=t$, and (2.29) is identical to (2.27) in the F-model. Thus the F- and S-branches with $f(0, t)=1$ are identical.

The S-branch of (2.29) with $f(0, t)=M_{\mathrm{c}}(t)$ has a vertical line segment at $x_{1}$, determined by $(\partial x / \partial f)_{t}=0$ or by $(\partial x / \partial s)_{t}=1-t u^{\prime}(s)=0$. The solution of this equation is $s_{\mathrm{c}}$, and corresponds to $f\left(x_{1}, t\right)=u\left(s_{\mathrm{c}}\right)=u\left[x_{1}+t u\left(s_{\mathrm{c}}\right)-T_{\mathrm{c}}\right]$. Thus $x_{1}=s_{\mathrm{c}}-t u s\left(s_{\mathrm{c}}\right)+T_{\mathrm{c}}$. On the other hand, it follows from $(2.22 a)$ that $f(0, t)=M_{\mathrm{c}}(t)=u\left(s_{\mathrm{c}}\right)$, so that $x_{1}=0$, and

$$
T_{\mathrm{c}} \equiv \int_{0}^{t} \mathrm{~d} \tau M_{\mathrm{c}}(\tau)=t u\left(s_{\mathrm{c}}\right)-s_{\mathrm{c}}
$$


Consequently, the second S-branch of $f(x, t)$ is obtained by shifting the curve of $f(x, t)$ by an amount $T_{\mathrm{c}}-t f=-t(f-1)+\left(T_{\mathrm{c}}-\mathrm{t}\right)$ (see figures $1(b)$ and $\left.2(b)\right)$. The first shift, $-t(f-1)$, transforms the initial value $u(x)$ into the two $F$-branches. The second shift is a parallel displacement of the F-branch with $f(0, t) \neq 1$ over a distance $\left(T_{\mathrm{c}}-t\right)$, such that the resulting S-branch becomes tangent to the $f$ axis at $M_{\mathrm{c}}$. In the S-model the branch marked $S_{1}$ in figures $1(b)$ and $2(b)$ is the physically relevant one with $f(0, t)=$ $M(t)$, since it determines the $c_{k}(t)$ through its behaviour at $x=-\infty$. The moments are determined by the values of the derivatives of the S-branch at $x=0$ corresponding to $s(0, t)=\eta$, defined in (2.23). The moments $M_{n}(t)$ with $n \geqslant 2$ only exist in the sol phase $\left(t<t_{\mathrm{c}}\right)$, and are divergent in the gel phase $\left(t \geqslant t_{\mathrm{c}}\right)$.

As $t \rightarrow \infty, M \rightarrow 0$ in both models, and the physical branch of $f(x, t)$ collapses towards the $x$ axis.

The initial conditions considered so far, i.e. $u\left(s_{0}\right) \rightarrow \infty$ or $u^{\prime}\left(s_{0}\right) \rightarrow \infty$ with $s_{0}>0$, guarantee the existence of two solutions for all times to each of the equations (2.12) and (2.20). Consider now initial distributions

$$
c_{k}(0) \sim k^{-n} \quad(k \rightarrow \infty) .
$$

Then $u(x)$ is only defined for $x \leqslant 0$.

Since $M$ is finite we must have $n>2$. If we further require $u^{\prime}(0)=M_{2}(0)=t_{\mathrm{c}}^{-1}$ to be finite, we must have $n>3$. The evolution of $f$, described by (2.27) or (2.29), is essentially the same as discussed above, but the curves stop at $f=1$. The graphical analysis is qualitatively the same as in figures $1,2,3$, provided all curves above the line $f=1$ are deleted. Consequently, the points $x_{0}, s_{\mathrm{b}}$ and $s_{\mathrm{c}}$ do not exist for $t<t_{\mathrm{c}}$ (see figure 1), and the solution of the kinetic equation has only one branch $\left(F_{1}\right.$ or $\left.S_{1}\right)$. For $t>t_{\mathrm{c}}$ the points $x_{0}, s_{\mathrm{b}}$ and $s_{\mathrm{c}}$ exist and a second branch appears. The case $2<n \leqslant 3$ where $M_{2}(0)=u^{\prime}(0) \rightarrow \infty$ corresponds to instantaneous gelation $\left(t_{\mathrm{c}}=0\right)$, and no further transition occurs.

\subsection{Further properties and examples}

Here we investigate the behaviour of the sol mass and gel fraction as $t \rightarrow t_{\mathrm{c}}$ and $t \rightarrow \infty$, and consider some special examples.

The behaviour of the gel fraction in the vicinity of the gel point $\left(t \downarrow t_{c}\right)$ is summarised in table 1 for two typical examples of short- and long-range initial distributions, characterised by the constants $m$ and $\Lambda$ and the exponent $\lambda$. We note that the critical exponent $\beta$, defined in (1.11), has the classical value $\beta=1$ in the F- and S-models for all initial distributions with $M_{3}(0)<\infty$. However, as soon as $M_{3}(0) \rightarrow \infty$, the exponent $\beta$ has a non-universal value, depending on the initial distribution, as shown in table 1 .

At long times the sol mass in the F-model, as determined by (2.17), exhibits exponential decay, i.e.

$$
M=c_{1}(0) \mathrm{e}^{-t}+\left[c_{1}^{2}(0) t+2 c_{2}(0)\right] \mathrm{e}^{-2 t}+\ldots
$$

since $u(x) \approx c_{1}(0) \mathrm{e}^{x}$ as $x \rightarrow-\infty$. In the S-model, however, the decay is algebraic, i.e. for $t \rightarrow \infty$

$$
M \simeq t^{-1}-2 c_{2}(0) / c_{1}^{2}(0) t^{2}+\ldots
$$

as follows from (2.22). 
Table 1. Behaviour of moments near $t_{c}$. For two classes of initial distributions: short range (case I, all $M_{n}(0)$ finite) and long range (case II, $c_{k}(0) \sim k^{-\lambda-2}$ for $k \rightarrow \infty$, corresponding to a small- $x$ singularity $(-x)^{\lambda}$ in $\left.u(x)\right)$, the table shows the behaviour of $s_{\mathrm{b}}((2.12))$, $s_{\mathrm{c}}((2.22 b))$ and $x_{0}((4.5 b))$ and the moments $M_{n}(t)(n=0,1,2,3$, see $(1.6))$ for $\theta=$ $\left(t-t_{c}\right) / t_{c} \rightarrow 0$ in the sol $\left(t<t_{c}\right)$ and gel $\left(t>t_{c}\right)$ phase for the F-and S-models. The symbol (?) indicates that the corresponding quantity is undefined.

\begin{tabular}{|c|c|c|c|c|c|c|}
\hline Case & \multicolumn{6}{|c|}{ Initial distribution } \\
\hline I & \multicolumn{6}{|c|}{$\begin{array}{l}\text { Short range: } M_{n}(0)<\infty ; M_{2}(0)=1 / t_{c} ; M_{3}(0)=m / t_{c}^{2} \\
u(x)=\Sigma k c_{k}(0) e^{k x}=1+x / t_{c}+\frac{1}{2} m\left(x / t_{c}\right)^{2} \quad(x \uparrow 0)\end{array}$} \\
\hline II & \multicolumn{6}{|c|}{$\begin{array}{l}\text { Long range: } M_{3}(0) \rightarrow \infty ; 1<\lambda<2 \\
u(x) \simeq 1+x / t_{\mathrm{c}}+\Lambda\left(-x / t_{\mathrm{c}}\right)^{\lambda} \quad(x \uparrow 0)\end{array}$} \\
\hline & \multicolumn{6}{|c|}{ Construction points $\mathrm{s}_{b}, s_{\mathrm{c}}$ and $x_{0}$ (figures 1,2 ) } \\
\hline I & \multirow{2}{*}{\multicolumn{6}{|c|}{$\begin{array}{ll}s_{\mathrm{b}}=-2 t_{\mathrm{c}} \theta / m & x_{0} \simeq t_{\mathrm{c}} \theta^{2} / 2 m \\
s_{\mathrm{c}}=-t_{\mathrm{c}} \theta / m & \theta=\left(t-t_{\mathrm{c}}\right) / t_{\mathrm{c}} \\
s_{\mathrm{b}}=-t_{\mathrm{c}}(\theta / \Lambda)^{1 /(\lambda-1)} \quad x_{0} \simeq t_{\mathrm{c}}\left(\frac{\lambda-1}{\lambda}\right)\left(\frac{\theta^{\lambda}}{\lambda . \Lambda}\right)^{1 /(\lambda-1)} \\
s_{\mathrm{c}}=-t_{\mathrm{c}}(\theta / \lambda \Lambda)^{1 /(\lambda-1)}\end{array}$}} \\
\hline II gel & & & & & & \\
\hline & \multicolumn{6}{|c|}{$\begin{array}{l}\text { Moments: } G=1-M \simeq B \theta^{\beta} \\
M_{2} \simeq C^{ \pm}|\theta|^{-\gamma} \quad M_{3} \simeq D^{ \pm}|\theta|^{-\gamma-1 / \sigma}\end{array}$} \\
\hline & $B$ & $\beta$ & $t_{\mathrm{c}} C^{ \pm}$ & $\gamma$ & $t_{\mathrm{c}}^{2} D^{ \pm}$ & $\sigma$ \\
\hline I sol & 0 & $?$ & 1 & 1 & $m$ & $\frac{1}{2}$ \\
\hline IF-gel & $2 / m$ & 1 & 1 & 1 & $m$ & $\frac{1}{2}$ \\
\hline I S-gel & $1 / m$ & 1 & $\infty$ & $?$ & $\infty$ & $?$ \\
\hline II sol & 0 & $?$ & 1 & 1 & $\infty$ & $?$ \\
\hline II F-gel & $\Lambda^{1 / 11-\lambda 1}$ & $\frac{1}{\lambda-1}$ & $\frac{1}{\lambda-1}$ & 1 & $\frac{\lambda \Lambda^{1 /(\lambda-1)}}{(\lambda-1)^{2}}$ & $\frac{\lambda-1}{\lambda}$ \\
\hline II S-gel & {$[\lambda .1]^{1 /(1-\lambda)}$} & $\frac{1}{\lambda-1}$ & $\infty$ & $?$ & $\infty$ & $?$ \\
\hline
\end{tabular}

The most important example corresponds to the monomer initial condition, $c_{k}(0)=$ $\delta_{k 1}$, where

$$
g(x, 0)=v(x)=\mathrm{e}^{x}, \quad f(x, 0)=u(x)=\mathrm{e}^{x},
$$

and where the gel point is $t_{\mathrm{c}}=1 / u^{\prime}(0)=1$. In this case the gel fraction in the F-model obeys a simple transcendental equation $(2.18)$ :

$$
G=1-\mathrm{e}^{-t G} \text {. }
$$

This equation has also been derived by Lushnikov (1978) using the master equation. In the S-model, on the other hand, the gel fraction is given by $(2.22)$ as

$$
G=1-t^{-1} \quad\left(t>t_{\mathrm{c}}\right) .
$$

In a similar way initial distributions of the form $c_{k}(0)=(1-d) \delta_{k l}+\frac{1}{2} d \delta_{k 2}$ can be analysed. Another example is one in which the initial mass distribution is exponential,

$$
k c_{k}(0)=a \mathrm{e}^{-k s_{\mathrm{n}}}, \quad a=\mathrm{e}^{s_{0}}-1 \quad\left(s_{0}>0\right),
$$


implying

$$
u(x)=a \mathrm{e}^{x-s_{0}} /\left(1-\mathrm{e}^{x-s_{0}}\right),
$$

where $a$ is determined through the normalisation $M(0)=u(0)=1$. Here $u(x)$ is only defined for $x<s_{0}$, and has the property $u\left(s_{0}\right) \rightarrow \infty$, guaranteeing that $(2.12)$ has two solutions for all $t>0$. Note that for $s_{0} \rightarrow \infty$ we recover the monodisperse initial condition. The gel point in this case is given as $t_{c}=\left[1-\exp \left(-s_{0}\right)\right]^{-1}$, illustrating that among all initial distributions the monomer one yields the largest gelation time.

The example may also be used to illustrate how the parametric representation $(2.22 a, b)$ can give a closed expression for $M(t)$ in the gel phase of the S-model, namely

$$
M(t)=\frac{1}{2} a\left[(1+4 / a t)^{1 / 2}-1\right] .
$$

Also note that $M(t) \simeq t^{-1}$ for $t \rightarrow \infty$, which is the same as for the monomer initial distribution. In figures 1 and 2 appear the intersection point $s_{b}(t)$, defined in (2.12), the tangent point $s_{\mathrm{c}}(t)$, defined in $(2.22 b)$ and the location $x_{0}(t)$ of the singularity in $f(x, t)$, defined in (2.28)). The behaviour of these points in the vicinity of $t_{c}$ is shown in table 1 for two typical classes of initial distribution. For the monomer case $s_{\mathrm{c}}$ and $x_{0}$ are given by

$$
s_{\mathrm{c}}=-\log t, \quad x_{0}=t-\log t-1,
$$

and similar expressions for the example in (2.38).

\section{Size distribution and moments}

\subsection{Lagrange's expansion for $c_{k}$}

In this section the size distribution and its moments will be calculated for a general initial distribution. We further study the asymptotic behaviour of $M_{n}(t)$ as $t \rightarrow t_{c}$ and $t \rightarrow \infty$.

Once the generating function $f(x, t)$ is known, the $c_{k}(t)$ can be found by expanding $f$ in powers of $z=\mathrm{e}^{x}$. Since the solution $(2.10 a, b)$ is given in parametric form, the desired expansion of $f$ can be obtained using Lagrange's expansion (see Abramowitz and Stegun 1974). For convenience we define

$$
\bar{f}(z, t)=f(x, t)=\sum_{k=1}^{\infty} k z^{k} c_{k}(t), \quad \bar{u}(z)=u(x)=\sum_{k=1}^{\infty} k z^{k} c_{k}(0),
$$

where $z=\mathrm{e}^{x}$. In this notation the general solution $(2.10 a, b)$ is given by

$$
z=y \exp [-t \bar{u}(y)+T], \quad \bar{f}=\bar{u}(y),
$$

where $y=\mathrm{e}^{s}$ and

$$
T=\int_{0}^{1} \mathrm{~d} \tau \mu(\tau)
$$

For any given (differentiable) $\bar{f}=\bar{u}(y)$ and $z(y)$, such that $z\left(y_{0}\right)=z_{0}$, Lagrange's expansion of $f$ in powers of $\left(z-z_{0}\right)$ is

$$
\bar{f}(z, t)=\bar{u}\left(y_{0}\right)+\sum_{k=1}^{\infty} \frac{\left(z-z_{0}\right)^{k}}{k !}\left[\left(\frac{\mathrm{d}}{\mathrm{d} y}\right)^{k-1} \tilde{u}^{\prime}(y)\left(\frac{y-y_{0}}{z(y)-z_{0}}\right)^{k}\right]_{y=y_{0}} .
$$


To calculate $c_{k}(t)$ one has to expand $\bar{f}(z, t)$ about $z_{0}=0$, where $y_{0}=\bar{u}\left(y_{0}\right)=0$. Thus we obtain from the preceding equations

$$
c_{k}(t)=\left(t k^{2} k !\right)^{-1} e^{-k T}\left([\mathrm{~d} / \mathrm{d} y)^{k} \mathrm{e}^{k t \bar{u}(y)}\right]_{y=0} .
$$

In the F-model $T=t$, and in the S-model $T=\int_{0}^{t} \mathrm{~d} \tau M(\tau)$, with $T=t$ in the sol phase and $T=T_{\mathrm{c}}$ in the gel phase by virtue of (2.24) and (2.30). Expression (3.5) gives a closed form solution for a given initial distribution, determined by $\bar{u}(z)$. In the F-model $c_{k}(t)$ has the same functional form in the sol and gel phases, whereas in the $S$-model the additional factor $\exp \left[-k\left(T_{\mathrm{c}}-t\right)\right]$ appears in the gel phase. For the first few $k$ one readily finds

$$
\begin{aligned}
& c_{1}(t)=c_{1}(0) \exp (-T), \quad c_{2}(t)=\left[c_{2}(0)+\frac{1}{2} t c_{1}^{2}(0)\right] \exp (-2 T), \\
& c_{3}(t)=\left[c_{3}(0)+2 t c_{2}(0) c_{1}(0)+\frac{1}{2} t^{2} c_{1}^{3}(0)\right] \exp (-3 T) .
\end{aligned}
$$

We note that such equations also follow from (2.1) directly, by solving iteratively for $c_{1}, c_{2}, \ldots$ Both methods rapidly become cumbersome.

As an example, where the size distribution for general $k$ can be calculated in closed form, we consider monodisperse initial conditions $(2.35)$ where $u(x)=\mathrm{e}^{x}$ and $\bar{u}(z)=z$. In the F-model, where $T=t$, one finds from (3.5) for all $t$

$$
c_{k}(t)=(k t)^{k-1} \mathrm{e}^{-k t} / k k !
$$

The same result has been obtained by Lushnikov (1978).

In the S-model, where $T=t$ in the sol phase, and $T=1+\log t$ in the gel phase $\left(M(t)=t^{-1}\right)$, the result is

$$
c_{k}(t)= \begin{cases}(k t)^{k-1} \mathrm{e}^{-k t} / k k !, & t<t_{\mathrm{c}}=1, \\ k^{k-2} \mathrm{e}^{-k} / t k !, & t>1 .\end{cases}
$$

The $c_{k}(t)$ are in both cases continuously differentiable across $t_{\mathrm{c}}$. Both results follow from Ziff and Stell's expressions for monomers of functionality $\bar{f}$ in the limit of large $\bar{f}$ with $\bar{f}^{2} t=\tau=$ finite. The result for the $F$-model has also been obtained by Lushnikov (1978) and that for the S-model by Leyvraz and Tschudi.

The large $k$ behaviour of $c_{k}$ and its behaviour for $t \rightarrow t_{\mathrm{c}}$ and $t \rightarrow \infty$ will be studied in $\$ \$ 4.4$ and 4.5 .

\subsection{Lagrange's expansion for $M_{n}$}

The moments of the size distribution can be derived, in a similar manner, from the general solution $f(x, t)=g_{x}$, using the expansion (2.5), i.e.

$$
f(x, t)=\sum_{n=0}^{\infty} M_{n+1} x^{n} / n !
$$

In this case we use the parametric representation $(2.10 a, b) \dagger$ with $x=x(s)$ and $f=u(s)$. We want to expand about $x=0$, corresponding to $s(0, t)=\zeta$ in the F-model (see $\$ 2.2$ ), and to $s(0, t)=\eta$ in the S-model (see $\$ 2.3$ ) for the physical branch of $f(x, t)$. Consider first the F-model, and apply Lagrange's expansion (3.4) with $y_{0} \rightarrow \zeta, z(y) \rightarrow$

$\div t$ is considered as a constant parameter. 
$x(s), z\left(y_{0}\right) \rightarrow x(\zeta)=0$ and $\bar{u}\left(y_{0}\right) \rightarrow u(\zeta)$. The result is

$$
\begin{aligned}
& M_{1}(t)=M(t)=u(\zeta), \\
& M_{n+1}(t)=\left[(\mathrm{d} / \mathrm{d} s)^{n-1} u^{\prime}(s)[1-t H(s)]^{-n}\right]_{s=\zeta} \quad(n \geqslant 1)
\end{aligned}
$$

with

$$
H(s)=[u(s)-u(\zeta)] /(s-\zeta)
$$

The first few moments read explicitly

$$
\begin{aligned}
& M_{2}(t)=u^{\prime}(\zeta)\left[1-t u^{\prime}(\zeta)\right]^{-1}, \quad M_{3}(t)=u^{\prime \prime}(\zeta)\left[1-t u^{\prime}(\zeta)\right]^{-3}, \\
& M_{4}(t)=u^{\prime \prime \prime}(\zeta)\left[1-t u^{\prime}(\zeta)\right]^{-4}+3 t\left[u^{\prime \prime}(\zeta)\right]^{2}\left[1-t u^{\prime}(\zeta)\right]^{-2} .
\end{aligned}
$$

The zeroth moment can be deduced from (2.6) and (2.11) as

$$
M_{0}(t)=v(\zeta)-\frac{1}{2} t u^{2}(\zeta)
$$

where $u(x)=v^{\prime}(x)$. The preceding equations give a closed form solution for $M_{n}(t)$ for a given initial condition $u(x)$ and for all times. The moments as given by (3.12), (3.13) are in fact the solutions of the moment equations. Away from the gel point (see discussion below (2.26)) these can be derived from the kinetic equation (2.1) with $\mu(t)=1$, by multiplying with $k^{n}$ and summing over all $k$, i.e.

$$
\begin{aligned}
& \dot{M}_{0}=-\frac{1}{2}+\frac{1}{2} G^{2}, \quad \dot{M}=-M_{2} G, \\
& \dot{M}_{n}=\frac{1}{2} \sum_{l=1}^{n-1}\left(\begin{array}{l}
n \\
l
\end{array}\right) M_{l+1} M_{n-l+1}-M_{n+1} G \quad(n \geqslant 2) .
\end{aligned}
$$

In the sol phase, where the gel fraction $G=1-M$ vanishes, the above equations reduce to the usual moment equations (see Drake 1972). In the gel phase, where $G \neq 0$, one can verify that (3.12)-(3.13) indeed satisfy (3.14). It is of interest to observe that Lushnikov (1978) also obtains the equation for $\dot{M}_{0}$ in $(3.14)$. In his approach the additional term, $\frac{1}{2} G^{2}$, in the gel phase originates from the fluctuations, which become of macroscopic size at and past the gel point in the limit of macroscopically large systems. In the sol phase, where the fluctuations behave normally, their contributions to $M_{0}$ vanish in the above limit of macroscopic systems.

Next, we turn to the S-model. Here, $\zeta$ in (3.10)-(3.13) should be replaced by $\eta$, defined in (2.23). In the sol phase $\eta=0$, and all results are identical to those in the F-model. In the gel phase, where $\eta=s_{c}$, the results are very different, since the denominators $\left[1-t u^{\prime}\left(s_{c}\right)\right]$ in $(3.10)-(3.12)$ are vanishing for all $t$ by virtue of $(2.22 b)$. Thus, $M_{n}(t) \rightarrow \infty$ for $n \geqslant 2$ and all $t \geqslant t_{\mathrm{c}}$, as is also clear from figure $2(b)$, where the physical branch has a vertical line segment at the point $\left(0, M_{c}\right)$. The total number of clusters, $M_{0}(t)$, given in (3.13), is still a well defined quantity in the gel phase, where it satisfies the moment equation

$$
\dot{M}_{0}=-\frac{1}{2} M^{2} \text {, }
$$

as can be verified using (3.13) (with $\zeta$ replaced by $\eta=s_{c}$ ) and $u\left(s_{c}\right)=M$.

As an example we consider again the monomer initial condition (2.35). Initially the moments are given by $M_{n+1}(0)=u^{(n)}(0)=1$. Since $v(s)=u(s)=\mathrm{e}^{s}$ we have $u^{(n)}(\zeta)=u(\zeta)=M$ (in the F-model), and $u^{(n)}(\eta)=u(\eta)=M$ (in the S-model), valid 
for all times. Consequently (3.12) and (3.13) lead to

$$
M_{0}=M\left(1-\frac{1}{2} t M\right), \quad M_{2}=M(1-t M)^{-1}, \quad M_{3}=M(1-t M)^{-3} .
$$

In both models $M=1$ for $t<t_{c}=1$. For $t>1, M$ is the solution of the equation $M=\exp (t M-t)$ with $M<1$ in the F-model, and $M=t^{-1}$ in the S-model. In the latter model

$$
M_{0}=1 / 2 t
$$

and all $M_{n}$ with $n \geqslant 2$ are infinite.

\subsection{Asymptotic properties of $M_{n}(t)$}

In this subsection we mainly discuss the behaviour of $M_{n}(t)$ in the vicinity of the gel point, and investigate for which initial distributions the exponents and amplitude ratios are universal. We briefly indicate the long-time properties of $M_{n}(t)$. The results are summarised in table 1 . We start with the $F$-model, where $\zeta=0$ in the sol phase, and the coefficients in (3.12)-(3.13) are given by the initial moments $u^{(n)}(0)=M_{n+1}(0)$ and $v(0)=M_{0}(0)$. We have in particular, for $t<t_{\mathrm{c}}=1 / u^{\prime}(0)$ :

$$
\begin{aligned}
& M_{2}(t)=\left(t_{\mathrm{c}}-t\right)^{-1}, \quad M_{3}(t)=t_{\mathrm{c}}^{3} M_{3}(0)\left(t_{\mathrm{c}}-t\right)^{-3}, \\
& M_{0}(t)=M_{0}(0)-\frac{1}{2} t .
\end{aligned}
$$

As $t \uparrow t_{c}$ all $M_{n} \rightarrow \infty$ for $n \geqslant 2$. In the gel phase, where by virtue of $(2.14)-(2.18)$

$$
\zeta=s_{\mathrm{b}}=t\left[u\left(s_{\mathrm{b}}\right)-1\right]=-t G,
$$

all moments are finite, since the zero of the denominator in $(3.10)-(3.12)$ is located at $s_{c}$ (see $(2.22 b)$ ), which is different from $\zeta=s_{b}$. However, at $t \downarrow_{c}$, one sees in figure 2 that $s_{\mathrm{b}} \uparrow 0$. Hence the denominators approach zero, and $M_{n}\left(t_{\mathrm{c}}\right) \rightarrow \infty$ for $n \geqslant 2$. In particular, for initial distributions with $M_{3}(0) \equiv m t_{\mathrm{c}}^{-2}<\infty$ one finds for $t \downarrow t_{\mathrm{c}}$

$$
\begin{array}{ll}
G(t)=1-M(t) \simeq 2\left(t-t_{\mathrm{c}}\right) / m t_{\mathrm{c}}, & M_{2}(t)=\frac{u^{\prime}(-t G)}{1-t u^{\prime}(-t G)} \simeq \frac{1}{t-t_{\mathrm{c}}}, \\
M_{3}(t)=\frac{u^{\prime \prime}(-t G)}{\left[1-t u^{\prime}(-t G)\right]^{3}} \approx \frac{m t_{\mathrm{c}}}{\left(t-t_{\mathrm{c}}\right)^{3}}, & M_{0}(t) \simeq M_{0}(0)-\frac{1}{2} t+2\left(t-t_{\mathrm{c}}\right)^{3} / 3 m^{2} t_{\mathrm{c}}^{2} .
\end{array}
$$

The last equality may be derived by expanding (3.13). It can be derived in a simpler way by integrating $\dot{M}_{0}$ in (3.14). Using the definitions of critical exponents and amplitudes in (1.13), we identify from (3.18) and (3.20): $C^{+}=C^{-}=1 / t_{\mathrm{c}}$ with $\gamma=1$, and $D^{+}=D^{-}=m / t_{\mathrm{c}}^{2}$ with $\sigma=1 / 2$. By comparison of (3.18) and (3.20) one sees that the mean cluster number $M_{0}(t)$ has a jump in its third derivative at $t_{c}$, i.e.

$$
\Delta M_{0}(t) \simeq M_{0}^{-}(t)-M_{0}^{+}(t) \simeq\left(2 t_{\mathrm{c}} / 3 m^{2}\right) \theta^{3},
$$

where $M_{0}^{+}(t)$ is an extrapolation into the gel phase of the function (3.18) for $M_{0}(t)$ in the sol phase; $\boldsymbol{M}_{0}^{-}(t)$ is the function (3.20) for the mean cluster number $\boldsymbol{M}_{0}(t)$ in the gel phase, and $\theta=t / t_{\mathrm{c}}-1$. Thus, the critical exponent $\alpha$ in (1.12) has the value $\alpha=-1$. However, in the case of integer non-positive $\alpha$ the amplitudes $A^{+}$and $A^{-}$ cannot be identified separately, because the singular terms in $M_{0}(t)$ are confluent with 
regular terms, and it is only meaningful to compare $\Delta M_{0}$ in (3.21) with $\Delta M_{0}=$ $\left(A^{+}+A^{-}\right) \theta^{3}$ in (1.12), yielding $A^{+}+A^{-}=2 t_{c} / 3 m^{2}$. Thus, the critical exponents $\alpha, \gamma$ and $\sigma$ have the classical values (1.17). One easily verifies that the ratios of critical amplitudes also have their classical values (1.18). Consequently, for all initial distributions $^{\dagger}$ with $M_{3}(0)<\infty$, exponents and ratios have universal (classical) values.

Next, we consider long-range initial distributions, in which $c_{k}(0) \sim k^{-\lambda-2}(k \rightarrow \infty)$ has an algebraic tail with $1<\lambda<2$. It is introduced through the generating function (2.7),

$$
u(x) \simeq 1+x / t_{\mathrm{c}}+\Lambda\left(-x / t_{\mathrm{c}}\right)^{\lambda} \quad(x \uparrow 0),
$$

which contains the parameters $\lambda$ and $\Lambda$ (for relations between small- $x$ singularities in $u(x)$ and algebraic tails in $c_{k}(0)$, see $\left.(4.13)\right)$.

As an illustration we calculate $M_{2}(t)$ from the expression (3.12), containing $u^{\prime}(\zeta)$ with $\zeta$ defined in (2.14). In the sol phase where $\zeta=0$ and $u^{\prime}(0)=1 / t_{\mathrm{c}}$ we find $M_{2}(t)=1 / t_{c}|\theta|$. Hence the $M_{2}$ amplitude, defined in (1.13), is $C^{+}=1 / t_{\mathrm{c}}$. In the gel phase we first determine $\zeta=s_{\mathrm{b}}$ (see (2.14)) by solving (2.12) for small $\zeta$ (since $t \downarrow t_{\mathrm{c}}$ ), with the result $\zeta=-t_{\mathrm{c}}(\theta / \Lambda)^{1 /(\lambda-1)}$ for $\theta \downarrow 0$. The last expression is inserted in (3.12) and yields to dominant order $M_{2}(t) \approx 1 /\left[(\lambda-1) t_{c} \theta\right]$. Hence, the $M_{2}$ amplitude in the gel phase is $C^{-}=1 /\left[t_{c}(\lambda-1)\right]$ and the ratio $C^{+} / C^{-}=\lambda-1$ does not have the universal value 1 of (1.18), but depends on the parameters $\lambda$ occurring in the initial distribution.

Similar calculations (for long-range initial distributions) of all moments $M_{n}(t)$ $(n=0,1,2,3)$ in the vicinity of the gel point have been performed for the $\mathrm{F}$ - and $\mathrm{S}$-models, and the results are summarised in table 1 (case II). In the gel phase of the F-model $M_{3}(t)$ approaches a form like (1.13) with a non-universal exponent $\sigma=$ $(\lambda-1) / \lambda$, and the amplitude $D^{+}$of $M_{3}(t)$ below the gel point is not well defined here, since the initial tail distribution remains until the gel point, as we shall see in $\S 4.3$. The mean cluster number, which can be calculated from (3.12) and table 1 , yields a non-universal exponent $\alpha=(\lambda-3) /(\lambda-1)$ and a non-universal value for the ratio $\left(A^{+}+A^{-}\right) C^{+} / B^{2}$.

In the S-model, where $M_{2}$ and $M_{3}$ are divergent for $t \geqslant t_{c}$, the amplitudes $C^{-}$and $D^{-}$are not well defined, and the amplitude ratios do not exist. The mean cluster number $M_{0}(t)$ behaves differently from the F-model. For initial distributions with $M_{3}(0) \equiv m / t_{c}^{2}<\infty$ as $t \downarrow t_{c}$ one finds

$$
M_{0}(t) \simeq M_{0}(0)-\frac{1}{2} t+\left(t-t_{c}\right)^{2} / 2 m t_{c}
$$

which should be compared with (3.20) in the F-model. Here the exponent, $\alpha=0$, although still universal for the class of initial distributions considered, is different from the classical value $\alpha=-1$ for bond percolation. The amplitude ratio will also be different from the classical value in (1.18).

At large times one finds from (3.12)-(3.13) for the F-model

$$
M_{0}(t) \simeq M_{2}(t) \simeq M_{3}(t) \simeq c_{1}(0) \mathrm{e}^{-t},
$$

and in the S-model (where $M_{n}(t)$ with $n \geqslant 2$ is not well defined in the gel phase)

$$
M_{0}(t) \simeq(2 t)^{-1} \text {. }
$$

$\uparrow$ For short range initial distributions $M_{n}(0)<\infty$ for all $n$. 


\section{Asymptotic properties of $c_{k}(t)$}

\subsection{Saddle point method}

In this section we derive asymptotic properties of $c_{k}(t)$. First the behaviour at fixed $t$ and $k \rightarrow \infty$ will be considered using the saddle point method or the moving singularity method, at least for short-range initial distributions. For long-range initial distributions we need the method of stuck singularities to calculate the large $k$ behaviour of $c_{k}(t)$. Secondly, we investigate a coupled (scaling) limit for $k \rightarrow \infty$ and $t \rightarrow t_{\mathrm{c}}$; thirdly we consider the limit $t \rightarrow \infty$ and $k$ fixed.

In order to obtain the large $k$ behaviour of $c_{k}(t)$ we write (3.5) as a contour integral:

$$
c_{k}(t)=\frac{\mathrm{e}^{-k T}}{t k^{2}} I_{k} \equiv \frac{\mathrm{e}^{-k T}}{t k^{2}} \oint \frac{\mathrm{d} z}{2 \pi \mathrm{i}} \frac{\mathrm{e}^{k(u \bar{u}(z)}}{z^{k+1}},
$$

where the path of integration is a closed contour around the origin. Choosing for the contour a circle of radius $\mathrm{e}^{x_{1}}$ and substituting $z=\mathrm{e}^{x}$ and $\bar{u}(z)=u(x)$ (see (3.1)), the integral becomes

$$
I_{k}=(2 \pi \mathrm{i})^{-1} \int_{x_{1}-\mathrm{i} \pi}^{x_{1}+\mathrm{i} \pi} \mathrm{d} x \exp [k(t u(x)-x)]
$$

where $x=x_{1}+\mathrm{i} x_{2}$. To obtain an asymptotic expression for $I_{k}$ by the saddle point method (which is a standard tool in analysing the solutions of the coagulation equation (Drake 1972)), we choose $x_{1}$ such that $F(x) \equiv t u(x)-x$ is at a maximum when the contour-the straight line between the two integration limits-crosses the real axis. Thus, $x_{1}$ is determined by $F^{\prime}\left(x_{1}\right)=t u^{\prime}\left(x_{1}\right)-1=0$, i.e. $x_{1}=s_{\mathrm{c}}$ as defined in $(2.22 b)$. Letting $x=s_{\mathrm{c}}+\mathrm{i} x_{2}$, the integral $(4.2)$ becomes

$$
I_{k}=(2 \pi)^{-1} \int_{-\pi}^{\pi} \mathrm{d} x_{2} \exp \left[k F\left(s_{\mathrm{c}}+\mathrm{i} x_{2}\right)\right] .
$$

Expanding $F$ about $s_{\mathrm{c}}$ and introducing $y=x_{2}$, we find that $I_{k}$ for large $k$ is approximated by

$I_{k}=(2 \pi)^{-1} \int_{-\infty}^{\infty} \mathrm{d} y \exp \left[k F\left(s_{\mathrm{c}}\right)-\frac{1}{2} k y^{2} F^{\prime \prime}\left(s_{\mathrm{c}}\right)\right] \simeq\left(2 \pi k t u^{\prime \prime}\left(s_{\mathrm{c}}\right)\right)^{-1 / 2} \exp \left(k T_{\mathrm{c}}\right)$,

where $F\left(s_{\mathrm{c}}\right)=T_{\mathrm{c}}$ on account of $(2.31)$. Thus we find for $k \rightarrow \infty$

$$
c_{k}(t) \simeq k^{-5 / 2}\left[2 \pi t^{3} u^{\prime \prime}\left(s_{c}\right)\right]^{-1 / 2} \exp \left[k\left(T_{\mathrm{c}}-T\right)\right] .
$$

In the F-model

$$
T-T_{\mathrm{c}}=t-t u\left(s_{\mathrm{c}}\right)+s_{\mathrm{c}}=x_{0}, \quad\left(t^{3} u^{\prime \prime}\left(s_{\mathrm{c}}\right)\right)^{-1}=\ddot{x}_{0} .
$$

In the first equation of $(4.5 b)$ we used $(2.28)$; the second equation is obtained by differentiating the first one twice, and eliminating $\dot{s}_{\mathrm{c}}$ from $(2.22 b)$. Note that $u\left(s_{\mathrm{c}}\right)=M_{\mathrm{c}}(t)$ in the sol phase has no connection with the physical mass $M(t)=1$. In the S-model

$$
T-T_{\mathrm{c}}=\left\{\begin{array}{ll}
x_{0}, & t<t_{\mathrm{c}}, \\
0 & t>t_{\mathrm{c}},
\end{array} \quad\left(t^{3} u^{\prime \prime}\left(s_{\mathrm{c}}\right)\right)^{-1}= \begin{cases}\ddot{x}_{0}, & t<t_{\mathrm{c}}, \\
\dot{M}, & t>t_{\mathrm{c}} .\end{cases}\right.
$$

Here we used (3.3) with $\mu(t)=M(t)$. This implies for the gel phase $T=T_{\mathrm{c}}$, since $M=M_{\mathrm{c}}$ on account of $(2.24)$. 
As an example we consider again the monomer initial condition (2.35), where $u\left(s_{\mathrm{c}}\right)=M_{\mathrm{c}}(t)=1 / t$ by virtue of $(2.22)$, and $x_{0}=t-1-\log t$. The resulting expression for the F-model, valid for fixed $t$ and $k \rightarrow \infty$, is

$$
c_{k} \simeq(2 \pi)^{-1 / 2} k^{-5 / 2} t^{k-1} \exp [-k(t-1)]
$$

In the sol phase of the S-model (4.6) applies again, and in the gel phase we have for fixed $t>t_{\mathrm{c}}$ and $k \rightarrow \infty$

$$
c_{k} \simeq(2 \pi)^{-1 / 2} k^{-5 / 2} t^{-1}
$$

The above analysis can only be used if the function $F(x)$ in (4.3) has a saddle point, i.e. if $s_{\mathrm{c}}$ exist. The latter condition is satisfied for initial distributions $u(x)$, regular for $x<s_{0}\left(s_{0}>0\right)$ with $u^{\prime}\left(s_{0}\right) \rightarrow \infty$ (see $\left.\$ 2.3\right)$.

The asymptotic behaviour of $c_{k}(t)$ depends upon the behaviour of $u(x)$ around $s_{c}$, and on the location of the singularity on the physical branch of $f(x, t)$, i.e. on the location of the vertical line segment in figures 1,2 and 3 . In the F-model this singularity is located at $x_{0}$ for all $t$, and the general asymptotic solution $(4.5 a, b)$ has the following properties: the large- $k$ behaviour of $c_{k}$ is dominated by the exponential factor $\exp \left(-k_{0} x\right)$ except at the gel point where $x_{0}=0$ and $c_{k}\left(t_{c}\right) \sim k^{-5 / 2}$. The transition from the exponential to $k^{-5 / 2}$ is accomplished as follows. Away from the gel point, we have $c_{k} \sim k^{-5 / 2} \exp \left(-k x_{0}\right)$ for $k \gg 1 / x_{0}$, while in the intermediate range $0 \ll k \ll 1 / x_{0}$ we have $c_{k} \sim k^{-5 / 2}$. As $t \rightarrow t_{c}, x_{0} \rightarrow 0$ and the $k^{-5 / 2}$ behaviour extends to infinity.

In the $S$-model the behaviour of $c_{k}(t)$ in the sol phase is identical to that in the F-model. In the gel phase the singularity on the physical branch of $f(x, t)$ is always located at the origin. Hence $c_{k} \sim k^{-5 / 2}$ for all $t \geqslant t_{\mathrm{c}}$.

\subsection{Moving singularity}

The asymptotic behaviour (4.5) of $c_{k}(t)$ can be derived directly from the generating function $(2.9 b)$ without using Lagrange's expansion and the subsequent saddle point method. We further investigate what can be learned about the asymptotics without explicitly solving the partial differential equation for the generating function.

Differentiating $(2.9 b)$ with respect to $x$ and solving for $f_{x}$ yields

$$
f_{x}=u^{\prime}(x+f t-T)\left[1-t u^{\prime}(x+f t-T)\right]^{-1} .
$$

Hence $f$ has a (moving) singularity in $x=x_{0}(t)$ (where $f_{x}$ becomes infinite), and $x_{0}(t)$ satisfies

$$
u^{\prime}\left(x_{0}+t f\left(x_{0}, t\right)-T\right)=1 / t .
$$

If the solution of $(2.22 b)$ exists, then one finds using $(2.10 a, b)$

$$
x_{0}(t)=s_{\mathrm{c}}-t u\left(s_{\mathrm{c}}\right)+T,
$$

identical to $x_{0}$ in (2.28). In the vicinity of $x_{0}(t)$ we try to represent $f$ as

$$
f(x, t)=f\left(x_{0}, t\right)-b\left(x_{0}-x\right)^{\lambda}
$$

where $b$ is positive, since $f(x, t)$ is increasing as $x \uparrow x_{0}$. By inserting the ansatz (4.11) into (4.8), and expanding the argument of $u^{\prime}$ around $s_{c}$, we readily obtain $\lambda=1 / 2$ 
and $b=\left[2 / t^{3} u^{\prime \prime}\left(s_{c}\right)\right]^{1 / 2}$. In particular, at $t_{c}$, where $x_{0}=s_{c}=0$, and $f\left(x_{0}, t_{c}\right)=1$ we have for $x \uparrow 0$

$$
f\left(x, t_{\mathrm{c}}\right) \simeq 1-\left(-2 x / m t_{\mathrm{c}}\right)^{1 / 2}
$$

where the relation $u^{\prime \prime}(0)=M_{3}(0)=m / t_{c}^{3}$ has been used.

The singularity (4.11) in $f(x, t)$ yields indeed the asymptotic result (4.5), as can be verified from the following relation, derived by Hendriks et al (1983): if

$$
\sum n_{k} \mathrm{e}^{k x} \simeq b\left(x_{0}-x\right)^{\lambda} \quad\left(x \uparrow x_{0}\right)
$$

then

$$
n_{k} \simeq(b / \Gamma(-\lambda)) k^{-\lambda-1} \exp \left(-k x_{0}\right) \quad(k \rightarrow \infty) .
$$

More limited information on the singularities in $f(x, t)$ can be obtained directly from the differential equation (2.8) without solving it explicitly:

$$
f_{r}=f_{x}(f-\mu) \text {. }
$$

We try to represent singular solutions in the vicinity of the singularity as

$$
f(x, t)=a-b\left(x_{0}-x\right)^{\wedge}+\ldots,
$$

where $a(t), b(t)$ and $x_{0}(t)$ (moving singularity) are unknown positive functions of $t$. For $\lambda<1,(4.15)$ is a consistent solution of $(4.14)$ if

$$
\dot{x}_{0}=\mu-a, \quad \dot{a}=-\frac{1}{2} b^{2}, \quad \lambda=\frac{1}{2} .
$$

In the F-model, where $\mu=1$, we have

$$
\dot{a}=1-\dot{x}_{0}, \quad b=\left(2 \ddot{x}_{0}\right)^{1 / 2} \text {. }
$$

A solution with $\dot{x}_{0}=0$ does not exist, as it implies $b=0$. With the help of (4.13) we obtain the following asymptotic expression as $k \rightarrow \infty$ :

$$
c_{k}(t) \simeq\left(\ddot{x}_{0} / 2 \pi\right)^{1 / 2} k^{-5 / 2} \exp \left(-k x_{0}\right) .
$$

The result is in agreement with $(4.5 a, b)$. However, one needs to solve (4.14) to obtain $x_{0}(t)$ explicitly. In the S-model, where $\mu(t)=M(t)$, we find in the sol phase again (4.18). There exists also a second solution with

$$
x_{0}=0, \quad a=M, \quad b=(-2 \dot{M})^{1 / 2},
$$

provided $\dot{M} \neq 0$ (gel phase). It yields for $k \rightarrow \infty$

$$
c_{k}(t) \simeq(-\dot{M} / 2 \pi)^{1 / 2} k^{-5 / 2}
$$

in agreement with $(4.5 a, c)$. However, in the present analysis $M(t)$ is an unknown function of $t$, to be determined by solving (4.14). Notice, however, that here we do not have any a priori reasons to exclude $x_{0} \neq 0$ in the gel phase.

\subsection{Stuck singularity}

The general asymptotic solutions (4.5), (4.18) and (4.20) in terms of a moving singularity at $x_{0}(t)$ are only valid as long as $s_{c}$ in $(2.22 b)$ can be found. If $u(x)$ is regular for $x<s_{0}\left(s_{0}>0\right)$ and $u^{\prime}\left(s_{0}\right)<\infty$, then $s_{\mathrm{c}}$ does not exist for $t<1 / u^{\prime}\left(s_{0}\right)$ (see below (2.22)), and the singularity is stuck at $s_{0}$ for $t \leqslant 1 / u^{\prime}\left(s_{0}\right)$, as can be simply understood from the graphical construction in figure $1(a)$. In this category the most 
singular class of initial distributions is one in which $u(x)$ has a singularity at $s_{0}=0$, with $u^{\prime}(0)=1 / t_{c}<\infty^{\dagger}$, as given by (3.22).

An explicit example of a long-range initial distribution is given through the generating function

$u(x)=\sum_{k=1}^{\infty} k c_{k}(0) \mathrm{e}^{k x}=-1+2 \mathrm{e}^{x}+\left(1-\mathrm{e}^{x}\right)^{3 / 2}, \quad \bar{u}(z)=-1+2 z+(1-z)^{3 / 2}$,

where the parameters in (3.22) have the values $t_{c}=\frac{1}{2}, \lambda=\frac{3}{2}$ and $\Lambda=1$. The size distribution follows from the binomial formula as

$$
c_{1}(0)=1 / 2, \quad c_{k}(0)=(3 / 4 \sqrt{\pi}) \Gamma(k-3 / 2) / k k ! \quad(k \geqslant 2),(4.21 b)
$$

and has an algebraic tail $c_{k}(0) \simeq(3 / 4 \sqrt{\pi}) k^{-7 / 2}(k \rightarrow \infty)$, in agreement with (4.13). The exact solution $c_{k}(t)$ for this example is given in its most explicit form by the contour integral (4.1) combined with $\tilde{u}(z)$ in $(4.21 a)$.

In order to determine its large- $k$ behaviour it is more convenient to use the generating function, and we shall now proceed to do so for the more general long-range initial distribution, defined through (3.22).

Here the singularity is stuck at $x=0$ for $t \leqslant t_{c}$ (sol phase). The behaviour of $f=u(x+t f-t)$ close to the stuck singularity can be obtained by expanding the solution for small argument with the result, valid for $x \uparrow 0$ and $t \leqslant t_{\mathrm{c}}$,

$$
f \approx 1+[x+t(f-1)] / t_{\mathrm{c}}+\Lambda\left\{[-x-t(f-1)] / t_{\mathrm{c}}\right\}^{\lambda} .
$$

For $t<t_{\mathrm{c}}$ one finds

$$
f(x, t) \simeq 1+x /\left(t_{\mathrm{c}}-t\right)+\left(-x / t_{\mathrm{c}}\right)^{\lambda}\left(\Lambda /|\theta|^{\lambda+1}\right),
$$

where $\theta=t / t_{\mathrm{c}}-1$. Thus at all fixed $t$ below $t_{\mathrm{c}}$ the algebraic tail, $c_{k}(t) \sim k^{-2-\lambda}$, remains, but it increases in strength with an amplitude $\left(1-t / t_{c}\right)^{-\lambda-1}$. The asymptotic expression for $c_{k}(t)$ is listed in table 2 .

Table 2. Behaviour of size distribution near $t_{\mathrm{c}}$. Properties of the size distribution in the scaling limit $\left(k \rightarrow \infty, \theta=\left(t-t_{\mathrm{c}}\right) / t_{\mathrm{c}} \rightarrow 0\right.$ with $k|\theta|^{1 / \sigma}=$ constant $)$ in the sol $(\theta<0)$ and gel $(\theta>0)$ phases for the $\mathrm{F}$ - and $\mathrm{S}$-models with initial distributions (case I, II) defined in table 1 . The symbol (?) indicates that the corresponding quantity is undefined.

\begin{tabular}{lllll}
\hline \multicolumn{5}{c}{$c_{k} \approx q_{0} k^{-\tau}\left[\exp -q_{1} k|\theta|^{1 / \sigma}\right]$} \\
\hline Case & $q_{0}$ & $q_{1}$ & $\tau$ & $\sigma$ \\
\hline I sol & $\left(2 \pi m t_{\mathrm{c}}\right)^{-1 / 2}$ & $t_{\mathrm{c}} / 2 m$ & $\frac{5}{2}$ & $\frac{1}{2}$ \\
I F-gel & $\left(2 \pi m t_{\mathrm{c}}\right)^{-1 / 2}$ & $t_{\mathrm{c}} / 2 m$ & $\frac{5}{2}$ & $\frac{1}{2}$ \\
I S-gel & idem & 0 & $\frac{5}{2}$ & $?$ \\
II sol & {$\left[\Lambda / \Gamma(-\lambda) t_{\mathrm{c}}^{\lambda}|\theta|^{\lambda+1}\right]$} & 0 & $2+\lambda$ & $?$ \\
II gel point & $-\left[\Gamma(-1 / \lambda)\left(\Lambda t_{c}\right)^{1 / \lambda}\right]^{-1}$ & 0 & $2+1 / \lambda$ & $?$ \\
II F-gel & {$[\theta / \Lambda \lambda]^{(1-\lambda / 2) /(\lambda-1)}$} & $t_{\mathrm{c}}\left(\frac{\lambda-1}{\lambda}\right)(\lambda \Lambda)^{1 /(1-\lambda)}$ & $\frac{5}{2}$ & $\frac{\lambda-1}{\lambda}$ \\
II S-gel & {$\left[2 \pi \lambda(\lambda-1) \Lambda t_{\mathrm{c}}\right]^{1 / 2}$} & 0 & $\frac{5}{2}$ & $?$ \\
\hline
\end{tabular}

\footnotetext{
$†$ Initial distributions with $u^{\prime}(0) \rightarrow \infty$ produce instantaneous gelation.
} 
At $t=t_{\mathrm{c}}$ the preceding analysis is not correct, since the terms in (4.22), linear in $(f-1)$, cancel. Here we find for $x \uparrow 0$

$$
f\left(x, t_{\mathrm{c}}\right) \simeq 1-\left(-x / \Lambda t_{\mathrm{c}}\right)^{1 / \lambda}
$$

which implies a different algebraic tail, $c_{k}\left(t_{c}\right) \sim k^{-2-1 / \lambda}$, at the gel point, the coefficient of which is given in table 2 .

For $t>t_{\mathrm{c}}$ the results of $\S \S 4.1$ and 4.2 can be applied, and (4.18) and (4.20) yield the tail distribution $c_{k} \sim k^{-5 / 2} \exp \left(-k x_{0}\right)$ in the F-model, and $c_{k} \sim k^{-5 / 2}$ in the S-model, valid for all initial distributions with $u^{\prime \prime}(0) \rightarrow \infty$ or $1<\lambda<2$ in (3.22).

For initial distributions of the form (3.22) with $\lambda>2$ (so that $u^{\prime \prime}(0)=m / t_{c}^{2}<\infty$ ), one verifies similarly that the algebraic tail, $c_{k}(t) \sim k^{-2-\lambda}$, remains for all fixed $t$ below $t_{\mathrm{c}}$. However, at $t=t_{\mathrm{c}}$ we find as $x \uparrow 0$

$$
f\left(x, t_{\mathrm{c}}\right) \simeq 1-\left(-2 x / m t_{\mathrm{c}}\right)^{1 / 2}+\mathrm{O}\left(|x|^{(\lambda-1) / 2}\right)
$$

with a leading singularity equal to (4.12), and not to the behaviour in (4.24).

Initial distributions of the form $c_{k}(0) \sim k^{-2-\lambda} \exp \left(-k s_{0}\right)$ with $s_{0}>0$ (where $u^{\prime}\left(s_{0}\right)<$ $\infty)$ produce stuck singularities and corresponding asymptotic behaviour of $c_{k}$ only for $t<1 / u^{\prime}\left(s_{0}\right)$, which is smaller than $t_{\mathrm{c}}$.

\subsection{Scaling properties near $t_{c}$}

An interesting limit is the scaling limit of the size distribution $c_{k}(t)$, in which $k \rightarrow \infty$ and $t \rightarrow t_{\mathrm{c}}$ with $\left|t-t_{\mathrm{c}}\right| k^{\sigma}$ kept fixed. The purpose of this subsection is to investigate for which class of initial distributions $c_{k}(t)$ has the scaling property $(1.16)$ in that limit.

For short-range $c_{k}(0)$ (case I of table 1 with all $M_{n}(0)<\infty$ ) the large- $k$ behaviour is given by (4.18) and (4.20), and we need the behaviour of $x_{0}(t)$ and $M(t)$ close to $t_{c}$, as has already been calculated in table 1 . The result for the F- and S-models in the sol phase and for the F-model in the gel phase is

$$
c_{k}(t) \simeq\left(2 \pi m t_{c}\right)^{-1 / 2} k^{-5 / 2} \exp \left[-k\left(t-t_{c}\right)^{2} / 2 m t_{c}\right],
$$

whereas the gel phase of the $\mathrm{S}$-model gives

$$
c_{k}(t) \simeq\left(2 \pi m t_{\mathrm{c}}\right)^{-1 / 2} k^{-5 / 2} .
$$

These expressions ${ }^{\dagger}$ only depend upon $t_{\mathrm{c}} \equiv 1 / M_{2}(0)$ and $m \equiv t_{\mathrm{c}}^{2} M_{3}(0)$. Note that the crossover between exponential and $k^{-5 / 2}$ behaviour occurs at $k=k_{\xi}$, where $k_{\xi}$ is the critical cluster size (1.14), behaving for $t \rightarrow t_{\mathrm{c}}$ as $k_{\xi} \simeq m t_{\mathrm{c}} /\left(t-t_{\mathrm{c}}\right)^{2}$. Thus, the scaling limit of $c_{k}(t)$ in the F-model gives the scaling form (1.16) with exponents $\tau=\frac{5}{2}, \sigma=\frac{1}{2}$, critical amplitude $q_{0}=\left(2 \pi m t_{c}\right)^{-1 / 2}$ and scaling function $\Phi(x)=\exp \left(-q_{1} x\right)$. The exponents and the amplitude ratio $C^{+} B / 4 \pi q_{0}^{2}=1$ have their classical values $(1.17)$ and (1.18) and are universal.

In the S-model this is only true in the sol phase. In the gel phase $c_{k}(t)$ in $(4.26 b)$ does not have the scaling form; the exponent $\sigma$ is not well defined; the amplitude ratio $C^{+} B / 4 \pi q_{0}^{2}=\frac{1}{2}$ does not have the classical value (1.18) for bond percolation, as discussed in $\S 1$, but exponent $\tau$ and amplitude ratio are universal. The results are summarised in table 2, together with those for the long-range initial distribution, denoted as case II in table 1 . The leading singularity in the generating function $f(x, 0)$

$\div$ For $c_{k}(0)=\delta_{k 1}$ one finds $t_{\mathrm{c}}=m=1$. 
for case II is of the form $\Lambda\left(-x / t_{\mathrm{c}}\right)^{\lambda}$ with $1<\lambda<2$, and the scaling property does not hold. If the results in the sol and gel phases (given in table 2), which are only valid at a fixed value of $t$ (with $t \neq t_{c}$ ), are extrapolated towards the gel point, one finds the following behaviour of $c_{k}(t)$ : as $t \uparrow t_{\mathrm{c}}$ the exponent $\tau=2+\lambda$, the critical amplitude $q_{0} \rightarrow \infty$ and $\sigma$ does not exist; as $t \downarrow t_{\mathrm{c}}$ one has $\tau=\frac{5}{2}, q_{0} \rightarrow 0$, and $\sigma$ does not exist in the S-model, whereas $\sigma=(\lambda-1) / \lambda$ in the F-model; at the gel point $\tau=2+1 / \lambda$ and $q_{0}$ has a finite non-vanishing value.

Consequently, universality does not hold for long-range initial distributions. Similar conclusions apply to long-range initial distributions with $\lambda>2$, as briefly discussed around (4.25).

We finally remark that the scaling limit and scaling property may be discussed equally well in terms of the generating functions $g(x, t)$ or $f(x, t)$, as has been done by Aharony (1980). Here we only mention the analogue (1.15) of the critical isotherm

$$
f\left(x, t_{\mathrm{c}}\right)=1-E(-x)^{1 / \delta},
$$

where $E$ is related to $q_{0}$ in (1.16) through $E=-q_{0} \Gamma(-1 / \delta)$, as follows directly from (4.13). By comparison of (4.27) with (4.12) and (4.24) the values for $\delta$ and $E$ can be identified. For initial distributions with $M_{3}(0)=m / t_{c}^{2}<\infty$ we find $\delta=2$ and $E=$ $\left(2 / m t_{\mathrm{c}}\right)^{1 / 2}$, yielding a universal and classical exponent, $\delta=2$, and amplitude ratio $C^{+} B / E^{2}=1$. For initial distributions (4.21) with $M_{3}(0) \rightarrow \infty$ we find from (4.24) that $\delta=\lambda$ and $E=\left(\Lambda t_{\mathrm{c}}\right)^{-1 / \lambda}$, and universality does not hold.

It is also of interest to investigate corrections to scaling. The scaling form (4.26) was obtained from the large- $k$ results $(4.4),(4.5)$ as the leading contribution if $\theta=t / t_{\mathrm{c}}-1$ approaches zero with $x=k|\theta|^{1 / \sigma} \mathrm{kept}$ fixed. It applies to short-range initial distributions defined in table 1 (case I). Inclusion of the next correction term in orders of $1 / k$ yields an asymptotic expression of the general form

$$
c_{k}(t) \simeq q_{0} k^{-\tau} \Phi(x)+k^{-\tau-\Omega} \Psi(x)+\ldots .
$$

As an illustration we consider only the sol phase $\left(t<t_{c}\right)$, but the results for the $F$ and S-models at and past the gel point can be obtained similarly. The first term on the right-hand side is the universal scaling form with $\tau=\frac{5}{2}$ and $\Phi(x)=\exp \left(-q_{1} x\right)$, already discussed above and listed in table 2 (case I). In the correction to scaling appear the new exponent $\Omega$, which equals $\frac{1}{2}$, and the function $\Psi(x)$, which has the general form $\sqrt{x}(a+b x) \exp \left(-q_{1} x\right)$ with constants $a$ and $b$. For the monodisperse initial condition the above results can be easily verified using (3.7).

In the case of long-range initial distributions (3.22) corrections to scaling can be calculated using the methods of $\$ 4.3$. In the sol phase $\left(t<t_{c}\right)$ the leading correction to $(4.23)$ gives

$f(x, t) \simeq 1+x / t_{c}|\theta|+\left(-x / t_{c}\right)^{\lambda}\left(\Lambda /|\theta|^{\lambda+1}\right)-\left(-x / t_{c}\right)^{2 \lambda-1}\left(\lambda \Lambda^{2} /|\theta|^{2 \lambda+1}\right)+\ldots$.

The term with $(-x)^{\lambda}$ yields with the help of (4.13) the non-universal behaviour (4.28) with $\tau=\lambda+2$ and $\Phi(x)=1$, as already discussed and listed in table 2 (case II). The term with $(-x)^{2 \lambda-1}$ yields through (4.13) the correction to scaling in (4.28) with $\Omega=\lambda-1$ and $\Psi(x)=$ constant.

\subsection{Long-time behaviour of $c_{k}(t)$}

The graphical analysis in figure 3 shows that the physical branch of $f(x, t)$ becomes small for large $t$, and can be obtained from a perturbation calculation. For the F-model 
we obtain from $(2.9 b)$ as $t \rightarrow \infty$

$$
f=u(x+t f-t) \simeq c_{1}(0) \mathrm{e}^{x+t f-t}+2 c_{2}(0) \mathrm{e}^{2 x+2 t f-2 t}+\ldots
$$

where we assume $c_{1}(0) \neq 0$. Solving for $\mathrm{e}^{x}$ yields

$$
\mathrm{e}^{x} \simeq\left(f / c_{1}(0)\right) \exp \left[t-f\left(t-2 c_{2}(0) / c_{1}^{2}(0)\right)\right]
$$

where the last term in the exponent is always small compared with $t$. Application of Lagrange's expansion finally yields for $t \rightarrow \infty$

$$
c_{k}(t) \simeq \frac{(t k)^{k-1} \mathrm{e}^{-k t}}{k k !}\left(c_{1}(0)\right)^{k}\left\{1+\frac{2 c_{2}(0)}{t c_{1}^{2}(0)}\right\}^{k-1} .
$$

The correction term in braces depends on the relative magnitude of $k$ and $t$. For fixed $k$ the relative correction is $\mathrm{O}(1 / t)$.

The size distribution in the S-model differs for $t>t_{\mathrm{c}}$ (gel phase) from that in the F-model by a factor exp $k\left(t-T_{\mathrm{c}}\right)$, and follows from (3.5). According to (2.31) and $(2.22 a)$ the exponent is

$$
\begin{aligned}
t-T_{\mathrm{c}} & =t-t u\left(s_{\mathrm{c}}\right)+s_{\mathrm{c}}=t-t M(t)+s_{\mathrm{c}} \\
& \simeq t-\log \left(c_{1}(0) t\right)-1-2 c_{2}(0) / c_{1}^{2}(0) t .
\end{aligned}
$$

The approximate equality $(4.33 b)$ is only valid for long times. It can be obtained from (2.34) and the relation

$$
s_{\mathrm{c}} \approx-\log \left(c_{1}(0) t\right)-4 c_{2}(0) / c_{1}^{2}(0) t+\ldots
$$

which is the long-time solution to $(2.22 b)$. The resulting expression for the long-time behaviour in the $\mathrm{S}$-model is

$$
c_{k}(t)=\frac{k^{k-2} \mathrm{e}^{-k}}{k ! t}\left(1+\frac{2 c_{2}(0)}{c_{1}^{2}(0) t}\right)^{k-1} \exp \left(-\frac{2 c_{2}(0) k}{c_{1}^{2}(0) t}\right) .
$$

Here the dominant term is independent of the initial distribution, and identical to the solution (3.8) for the monodisperse case. For fixed values of $k$ the leading correction is of relative order $1 / t$ and independent of $k$. For $k$-values proportional to $t$ the correction factors cancel, and the solution (3.8) for the monodisperse case is recovered.

\section{Summary}

On the basis of Smoluchowski's coagulation equation we discussed the kinetics of the gelation transition in systems of branched polymers, starting from arbitrary initial distributions, for a model in which no cyclisation is allowed.

The standard theories-Flory and Stockmayer's statistical theory of the most probable distribution, and the statistical mechanical treatment of random bond percolation on Bethe lattices or Cayley trees-can only assign equilibrium weights to the size distribution of polymers, corresponding in our kinetic description with the very special monomer initial distribution.

We presented two models (F-and S-models) that differ only in the gel phase. Which one of these non-equilibrium models is appropriate will depend on the experimental circumstances. In the F-model all reactive groups on both sol and gel are available for bonding of sol particles, whereas in the $\mathrm{S}$-model only the reactive 
groups on sol particles can form chemical bonds. The latter model corresponds to the situation in which the gel is continuously removed from the reacting polymer system (e.g. precipitation). The post-gelation solutions in the F- and S-models are respectively identical to Flory's and to Stockmayer's classical results in the gel phase.

The asymptotic form of the size distribution $c_{k}(t)$ for large $k$ and $t \rightarrow t_{c}$ depends only on the initial moments $M_{2}(0)$ and $M_{3}(0)$, at least for short-range initial distributions (i.e. with all $\left.M_{n}(0)<\infty\right)$. The dominant behaviour of $c_{k}(t)$ at large $t$ is determined by the initial concentrations of monomers and dimers, $c_{1}(0)$ and $c_{2}(0)$.

Concerning scaling and universality we have shown by calculating scaling functions, critical exponents and critical amplitude ratios that the F-model falls in the same universality class as the classical bond percolation problem on Cayley trees, provided the initial distribution is of short range. For long-range $c_{k}(0)$ (having algebraic tails) the form of $c_{k}(t)$ in the scaling limit $\left(k \rightarrow \infty, t \rightarrow t_{\mathrm{c}}\right.$, such that $k\left|t-t_{\mathrm{c}}\right|^{1 / \sigma}=$ constant $)$ depends on parameters of the initial distribution and is therefore non-universal (see $\S \$ 3.3$ and 4.4 ).

In the $\mathrm{S}$-model the scaling property does not hold in the gel phase, where $c_{k}(t) \simeq$ $q_{0} k^{-\tau}$. Here the 'susceptibility' $M_{2}$, and the critical cluster size $k_{\xi} \simeq M_{3} / M_{2}$ or correlation length $\xi$ remain infinite for $t \geqslant t_{\mathrm{c}}$, so that the exponents $\gamma$ and $\sigma$, and critical amplitudes $C^{-}$and $D^{-}$are undefined above $t_{\mathrm{c}}$. The 'specific heat' exponent $\alpha$, describing the singularity in the mean number of clusters $M_{0}$, has the value $\alpha=0$, and differs from the classical value $(\alpha=-1)$ bond percolation. Furthermore, none of the ratios of critical amplitudes in the $S$-model have the classical values (1.18) for bond percolation.

For short-range initial conditions the results for the S-model are independent of initial conditions. Thus the S-model falls into a different universality class from the classical bond percolation problem.

Recently (Herrmann et al 1982, 1983, Bansil et al 1983) several kinetic gelation models have been discussed that do not belong to the universality classes of random percolation or to the classical gelation theories (F-and S-models).

\section{Acknowledgment}

RMZ acknowledges the Office of Basic Energy Sciences, US Department of Commerce, for support of this research.

\section{References}

Abramowitz M and Stegun I 1974 Handbook of Mathematical Functions (New York: Dover) $\$ 3.6$ Aharony A 1980 Phys. Rev. B 22400

Bansil R, Herrmann H J and Stauffer D 1983 Macromolecules

Cohen R J and Benedek G B 1982 J. Phys. Chem. 863696

Donoghue E $1982 \mathrm{~J}$. Chem. Phys. 774236

Donoghue E and Gibbs J H 1979 J. Chem. Phys. 702346

Drake R L 1972 in Topics in Current Aerosol Research vol 3, part 2 eds G M Hidy and J R Brock (New York: Pergamon)

Dusek K 1979 Polymer Bulletin 1523

Ernst M H. Hendriks E M and Ziff R M 1982 J. Phys. A: Math. Gen. 15 L743

Falk $M$ and Thomas R E 1974 Can. J. Chem. 523285

Feller W 1968 An introduction to probability theory and its applications (New York: Wiley). 
Flory P J 1953 Principle of Polymer Chemistry (Ithaca, NY: Cornell UP) ch 9 de Gennes P G 1976 J. Physique Lett. L37 1

1979 Scaling concepts in polymers (Ithaca, NY: Cornell UP) ch V2 Hendriks E M, Ernst M H and Ziff R M 1983 J. Stat. Phys.

Herrmann H J, Landau D P and Stauffer D 1982 Phys. Rev. Lett. 49412

Herrmann H J, Stauffer D and Landau D P 1983 J. Phys. A: Math. Gen. 16 1221-40

van Kampen N G 1981 Stochastic Processes in Physics and Chemistry (Amsterdam: North-Holland) ch 9 Leyvraz F and Tschudi H R 1981 J. Phys. A: Marh. Gen. 143389

Lushnikov A A 1978 Izvestiya, Ocean. and Atmos. Phys. 14378

Lushnikov A A, Tokar Ya I, Tsitskishvili M S 1981 Dokl. Phys. Chem. 2561155

McLeod J B 1962 Q. J. Math. 13 119, 192, 283

von Schulthess G K, Benedek G B and DeBlois R W 1980 Macromolecules 13939

Schmidt M and Burchard W 1981 Macromolecules 141370

Stauffer D 1976 J. Chem. Soc. Faraday Trans. II 721354

Stauffer D, Coniglio A and Adam M 1982 in Polymer Networks ed K Dusek Adv. Polymer Sci. Vol 44 (Berlin: Springer) p 103

Stockmayer W H 1943 J. Chem. Phys. 1145

Ziff R M 1980 J. Stat. Phys. 23241

Ziff R M and Stell G $1980 \mathrm{~J}$. Chem. Phys. 733492

Ziman J 1979 Models of disorder (Cambridge: CUP) ch 7 